\title{
Estrategia pedagógica para desarrollar actitudes favorables en la enseñanza de la estadística en estudiantes de la licenciatura en Ciencias Ambientales
}

Pedagogical strategy to develop favorable attitudes in the teaching of statistics in students of the degree in Environmental Sciences

Estratégia pedagógica para desenvolver atitudes favoráveis ao ensino da estatística em alunos da licenciatura em Ciências Ambientais

Silberio García Sánchez

Universidad Autónoma de Guerrero, México silberio_garcia134@hotmail.com https://orcid.org/0000-0002-3641-3267

Alejandro Juárez Agis

Universidad Autónoma de Guerrero, México ajuarezagis@hotmail.com http://orcid.org/0000-0001-8839-112X

Branly Olivier Salome Universidad Autónoma de Guerrero, México branlyos@gmail.com https://orcid.org/0000-0003-0021-1767

Jacqueline Zeferino Torres Universidad Autónoma de Guerrero, México jackyezt@gmail.com https://orcid.org/0000-0001-5312-470X 


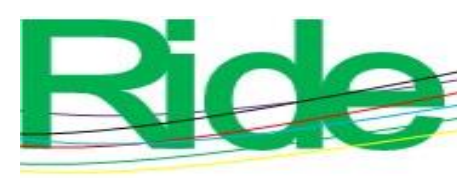

\section{Revista Iberoamericana para la Investigación y el Desarrollo Educativo ISSN $2007-7467$}

Mayra Rivas González

Universidad Autónoma de Guerrero, México

mrivasg@live.com.mx

https://orcid.org/0000-0002-2115-8152

* Autor de correspondencia: Alejandro Juárez Agis

\section{Resumen}

Las actitudes negativas hacia el aprendizaje de la estadística por parte de los estudiantes de nivel licenciatura influyen en el rendimiento de su aprendizaje. Estudiar estas actitudes hacia el aprendizaje de la estadística es de suma importancia para comprender el fenómeno. En ese sentido, el objetivo de la investigación fue analizar las actitudes hacia el aprendizaje y los conocimientos elementales de la estadística de estudiantes de la licenciatura en Ciencias Ambientales; con base en los resultados, se seleccionó y utilizó una estrategia pedagógica pertinente que orientó el proceso de aprendizaje en la unidad de aprendizaje de Estadística. El método utilizado fue de tipo mixto, pues se aplicó una encuesta cuantitativa (con escala tipo Likert), así como un instrumento de preguntas abiertas (parte cualitativa). Luego, se aplicó una propuesta de intervención para el desarrollo de actitudes positivas de los estudiantes hacia la estadística. El análisis de la información cuantitativa fue con el paquete estadístico SPSS 21.0. y Excel. Las preguntas abiertas se analizaron con base en el método de codificación y clasificación. En cuanto a los participantes, se llevó a cabo un muestreo no probabilístico por conveniencia e intencional a alumnos de la Escuela Superior de Ciencias Ambientales de la Universidad Autónoma de Guerrero.

Los resultados muestran que los estudiantes que llevaron el curso de estadística con la estrategia pedagógica implementada aprenden más en clases, pues se promovió el aprendizaje autónomo. Además, reconocieron la estrategia implementada como una herramienta que puede articularse en el desarrollo de cualquier unidad de aprendizaje. Asimismo, se evidenció motivación en ellos para el desarrollo de actitudes positivas hacia el aprendizaje de la estadística. Este tipo de iniciativas pedagógicas favorecen el pensamiento reflexivo de las actitudes hacia las estadísticas y ayudan a promover la construcción de conocimientos para intervenir y resolver problemas. Como conclusión, podemos decir que, en general, la percepción de los estudiantes frente a este tipo de estrategia fue muy buena; sin embargo, se deben tomar en cuenta otros factores que surgen en el aula (cansancio, pereza, etc.) para mejorar la ejecución de la estrategia. 


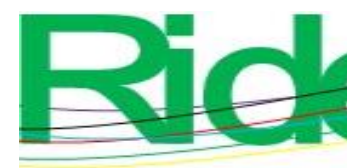

Revista Iberoamericana para la Investigación y el Desarrollo Educativo ISSN 2007 - 7467

Palabras clave: actitud del estudiante, estadística, método de aprendizaje.

\section{Abstract}

Negative attitudes towards the learning of statistics by undergraduate students influence the performance in their learning. Studying these attitudes towards learning statistics is of great importance so as to understand the phenomenon. In this sense, the objective of the research was to analyze the attitudes towards learning and the elementary knowledge of the statistics of students of the Degree in Environmental Sciences, based on the results, a pertinent pedagogical strategy was selected and used, the same which guided the process of learning in the Statistics Learning Unit. The method used was of a mixed type, the quantitative Likerttype survey was applied and in the qualitative part an instrument of open questions was used, in addition, an intervention proposal was made, for the development of positive attitudes of the students towards the statistics. The analysis of the quantitative information was done with the statistical package SPSS 21.0. and Excel, developing only descriptive analysis, for open questions, it was based on the method of coding, classification. Intentionally and for convenience a non-probabilistic sampling was carried out to students of the Higher School of Environmental Sciences of the Autonomous University of Guerrero.

The results show that the students who took the statistics course with the pedagogical strategy implemented learn more in class, promoting autonomous learning and recognized the strategy as the tool that can be articulated in the development of any learning unit. They also showed motivation for the development of positive attitudes towards learning statistics. On the other hand, they contribute to promote reflective thinking of attitudes towards statistics, having greater emphasis on the construction of knowledge that allows them to intervene and solve problems through this Project-Based Learning pedagogical strategy which was proposed. As a conclusion we can say, that the perception of the students working with this type of pedagogical strategy is really good, however; fatigue, laziness, etc in the classroom.), are some factors that must be taken into account, this would allow to have a better execution of the proposed pedagogical strategy.

Keywords: student attitude, statistics, learning method. 


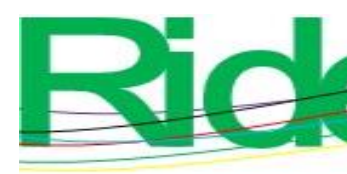

\section{Revista Iberoamericana para la Investigación y el Desarrollo Educativo ISSN 2007 - 7467}

\section{Resumo}

Atitudes negativas em relação à aprendizagem de estatística por alunos de graduação influenciam seu desempenho de aprendizagem. Estudar essas atitudes em relação ao aprendizado de estatísticas é de extrema importância para compreender o fenômeno. Nesse sentido, o objetivo da pesquisa foi analisar as atitudes frente à aprendizagem e conhecimentos elementares de estatística de alunos do curso de Ciências Ambientais; Com base nos resultados, foi selecionada e utilizada uma estratégia pedagógica pertinente que norteou o processo de aprendizagem na unidade de aprendizagem de Estatística. O método utilizado foi do tipo misto, uma vez que foi aplicada uma pesquisa quantitativa (com escala do tipo Likert), bem como um instrumento de questões abertas (parte qualitativa). Em seguida, foi aplicada uma proposta de intervenção para o desenvolvimento de atitudes positivas dos alunos em relação à estatística. A análise das informações quantitativas foi com o pacote estatístico SPSS 21.0. e Excel. As questões abertas foram analisadas com base no método de codificação e classificação. Em relação aos participantes, foi realizada uma amostragem não probabilística por conveniência e intencional para alunos da Escola Superior de Ciências Ambientais da Universidade Autônoma de Guerrero.

Os resultados mostram que os alunos que fizeram o curso de estatística com a estratégia pedagógica implementada aprendem mais em sala de aula, uma vez que foi promovida a aprendizagem autônoma. Além disso, reconheceram a estratégia implementada como uma ferramenta que pode ser articulada no desenvolvimento de qualquer unidade de aprendizagem. Da mesma forma, foi evidenciada neles motivação para o desenvolvimento de atitudes positivas em relação à aprendizagem estatística. Este tipo de iniciativas pedagógicas favorecem o pensamento reflexivo sobre as atitudes em relação à estatística e ajudam a promover a construção de conhecimentos para intervir e resolver problemas. Concluindo, podemos dizer que, em geral, a percepção dos alunos sobre este tipo de estratégia foi muito boa; entretanto, outros fatores que surgem em sala de aula (cansaço, preguiça, etc.) devem ser levados em consideração para melhorar a execução da estratégia.

Palavras-chave: atitude do aluno, estatística, método de aprendizagem.

Fecha Recepción: Abril 2020

Fecha Aceptación: Enero 2021 


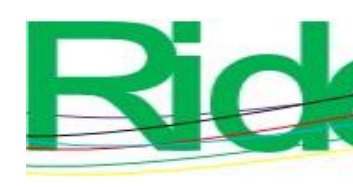

Revista Iberoamericana para la Investigación y el Desarrollo Educativo ISSN 2007 - 7467

\section{Introducción}

Todo trabajo de investigación parte de un problema al cual se busca ofrecer una propuesta de solución. En ese sentido, las investigaciones en educación estadística han considerado desde la década de los ochentas como objetivo principal el formar recursos humanos que usen apropiadamente el pensamiento estadístico (Schau, 2003). Sin embargo, hay factores afectivos que influyen de manera negativa en la construcción de conocimientos estadísticos, como los sentimientos, los valores y las actitudes hacia esta área o disciplina (Torres, Aparicio, Bazán y Abdounur, 2015).

Investigaciones realizadas por Cardoso, Cerecedo y Ramos (2012), Gómez-Chacón (2000) y Gil, Guerrero y Blanco (2006) señalan que algunas variables como el gusto por los números pueden influenciar muchas veces negativamente a los estudiantes. Asimismo, Gil et al. (2006) indican que ciertos estudiantes durante su vida académica manifiestan actitudes negativas hacia las matemáticas-estadísticas, lo que se convierte en una fuente de frustración, desánimo y angustia.

Por su parte, Comas, Martins, Nascimento y Estrada (2017) encontraron en una investigación realizada a universitarios que las actitudes en general son moderadas o positivas en los estudiantes, e indican que tener estudios previos en estadísticas incide en la actitud, aunque — como lo manifiestan Salinas y Mayén (2016) — la actitud global hacia la estadística empeora con los años de estudio.

En el estudio realizado por Salinas y Mayén (2016) en cinco centros escolares del Colegio de Ciencias y Humanidades de la Universidad Nacional Autónoma de México (UNAM) se indica que los estudiantes reconocen la importancia y utilidad de la estadística principalmente en su área de estudio, así como en la vida diaria, y señalan que la falta de estudios previos en esa área puede generar una actitud favorable hacia esta materia. Por su parte, Bautista, Morales, Dórame y Peralta (2016), en una muestra 392 estudiantes de diferentes carreras de la Universidad de Sonora, indican que la mayoría de estudiantes consultados asumen una actitud positiva hacia la estadística.

Asimismo, el trabajo de investigación realizado por Sánchez (2019) — con estudiantes de dos programas de licenciatura en Matemáticas, de la Universidad Autónoma de San Luis Potosí y de la Benemérita Universidad Autónoma de Zacatecas- hace referencia al factor utilidad, integrada por las preguntas 1, 6, 11, 16, 20 y 21 de la encuesta de Auzmendi. Este factor quedó integrado con las afirmaciones siguientes: considero la 


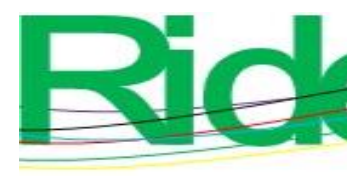

\section{Revista Iberoamericana para la Investigación y el Desarrollo Educativo ISSN 2007 - 7467}

estadística como una materia muy necesaria en la carrera, quiero llegar a tener un conocimiento más profundo de la estadística, saber utilizar la estadística incrementará mis posibilidades de trabajo, y para el desarrollo profesional de mi carrera una de las asignaturas más importantes que ha de estudiarse es la estadística. En pocas palabras, el referido autor encontró que la mayoría de los estudiantes tienen una actitud favorable hacia la estadística y muestran interés por aprenderla de forma exhaustiva.

Asimismo, para ese mismo autor, con el factor ansiedad se constituyen las preguntas 2, 7, 12, 17 y 22, las cuales son las siguientes: la asignatura de estadística se me da bastante mal, la estadística es una de las asignaturas que más temo, cuando me enfrento a un problema de estadística me siento incapaz de pensar con claridad, trabajar con la estadística hace que me sienta muy nervioso/a, y la estadística hace que me sienta incómodo/a y nervioso/a. Sánchez encontró que cerca de $30 \%$ de los estudiantes está en desacuerdo con la pregunta 2, y que la mayoría de los estudiantes no está de acuerdo con que la estadística sea una de las asignaturas más temidas (pregunta 7). Por otra parte, con la pregunta 12 la mayoría de la muestra dividió su respuesta de manera neutral y en desacuerdo, y la pregunta 17, en este caso, encontró que 1 de cada 2 estudiantes no se siente nervioso al trabajar con estadística.

De la misma manera, Sánchez (2019) encontró en su investigación en el factor confianza, que la mayoría de los estudiantes responde de manera neutral sobre la pregunta 8; para la pregunta 13, la mayoría de los estudiantes se sienten calmados al trabajar con un problema estadístico. Asimismo, para la pregunta 18 la mayoría está de acuerdo con esta pregunta.

Para el factor agrado, Sánchez (2019) encontró sobre la pregunta 4 una actitud desfavorable hacia la estadística. Con la pregunta 9 a los estudiantes en general no les divierte hablar con otros de estadística. Asimismo, para la pregunta 14, según Sánchez, se podría predecir una actitud desfavorable de los estudiantes hacia la estadística. Además, para la pregunta 19 resalta que 1 de cada 5 estudiantes está en desacuerdo con que le gustaría tener una ocupación en la que tuviera que utilizar estadística.

Por último, con el factor motivación, integrado por las preguntas 5, 10, 15, 20 y 25, Sánchez (2019) halló lo siguiente: la mayoría de los estudiantes considera que la estadística no es muy teórica para ser de utilidad, pero consideran que es útil para el profesional que no se dedique a la investigación, y se sienten motivados de utilizar la estadística en la vida profesional. 


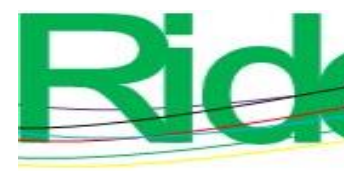

\section{Revista Iberoamericana para la Investigación y el Desarrollo Educativo ISSN 2007 - 7467}

En cuanto al factor ansiedad, en un estudio realizado por García, Escalera, Moreno y Santana (2016) a estudiantes del Centro de Estudios Tecnológicos Industrial y de Servicios n. ${ }^{\circ} 15$ en la Ciudad de Veracruz, se demostró que ese factor impide a los estudiantes alcanzar un buen rendimiento en el proceso de aprendizaje.

Por último, en la investigación de Rodríguez (2011) se indica que los estudiantes de carreras humanísticas poseen actitudes negativas hacia la estadística, mientras que los de carreras no humanísticas poseen actitudes positivas. Asimismo, se señala que influye la opinión sobre la utilidad en la profesión de los estudiantes, ya que es necesario explicarles la utilidad e importancia que tiene esta asignatura. Por ello, es necesario enseñar a los estudiantes herramientas estadísticas según la información que deban procesar en las investigaciones que desarrollen dentro del área de las ciencias ambientales.

Por lo anteriormente mencionado, el presente trabajo tuvo como finalidad analizar las actitudes hacia el aprendizaje y los conocimientos elementales de la estadística en estudiantes de la licenciatura en Ciencias Ambientales. Luego, con base en los resultados se seleccionó y utilizó una estrategia pedagógica pertinente para orientar el proceso y disminuir el rezago de aprendizaje en la unidad de aprendizaje Estadística de la Escuela Superior de Ciencias Ambientales de la Universidad Autónoma de Guerrero. Las preguntas que permitieron contestar el objetivo del estudio fueron las siguientes:

1. ¿Cómo evaluar al inicio y al final del curso el aprendizaje de los estudiantes de la licenciatura en Ciencias Ambientales para que tengan actitudes positivas hacia la unidad de aprendizaje de Estadística?

2. ¿Cómo evaluar el grado de compresión de los conocimientos elementales de estadística que tienen los estudiantes de la licenciatura en Ciencias Ambientales?

3. ¿Cómo pueden tener actitudes positivas hacia las estadísticas los estudiantes de la licenciatura en Ciencias Ambientales?

\section{Método}

En el presente trabajo de investigación se utilizó un método de tipo mixto (Hernández, Fernández y Baptista, 2014), pues se consideró que la combinación de técnicas cuantitativas y cualitativas sería lo más apropiado para responder al objetivo de la investigación. 


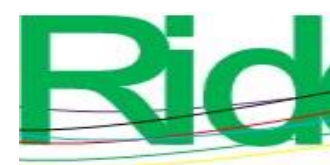

Revista Iberoamericana para la Investigación y el Desarrollo Educativo ISSN $2007-7467$

\section{Instrumentos de recogida de datos}

Instrumento cuantitativo

Para la parte cuantitativa de esta investigación se utilizó la encuesta cuantitativa de Auzmendi (1992), la cual estuvo constituida por 25 ítems que ofrecían 5 opciones según una escala de Likert $(1=$ totalmente en desacuerdo; $2=$ en desacuerdo; $3=$ neutral; $4=$ de acuerdo; 5 = totalmente de acuerdo). Primera encuesta.

Los factores medidos en los 25 ítems se enfocaron en utilidad (ítems 1, 6, 11, 20 y 21), ansiedad (2, 7, 12, 17 y 22), confianza (3, 8, 13, 18 y 23), agrado (4, 9, 14, 19 у 24$)$ y motivación $(5,10,15,20$ y 25).

En el factor utilidad se evaluó la importancia, conocimiento, satisfacción y dominio en estadística para el futuro profesional. En el factor ansiedad se valoró el temor, desconfianza e inseguridad hacia la estadística en su formación. El factor confianza evaluó la actitud positiva que se tiene hacia la estadística. El factor agrado se enfocó en el gusto por estudiar estadística. Por último, el factor motivación se centró en la actitud negativa (desinterés) hacia la estadística. A continuación, se detallan los enunciados que conformaron la encuesta de Auzmendi (tabla 1).

Así mismo, para obtener información sobre los aspectos cognitivos de los estudiantes después de llevar el curso se aplicó la encuesta de Ayuga, González, Grande y Martínez (2012), modificada para este trabajo y la contestaron 51 estudiantes (tercera encuesta). La cuarta encuesta fue sobre la percepción del uso de la estrategia pedagógica del ABP (Rodríguez y Cortés (2010). La contestaron 52 estudiantes. 


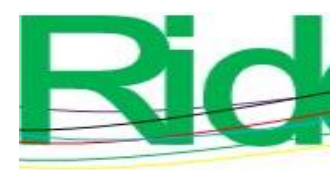

Revista Iberoamericana para la Investigación y el Desarrollo Educativo

ISSN 2007 - 7467

Tabla 1. Enunciados que conformaron el instrumento cuantitativo (encuesta de Auzmendi)

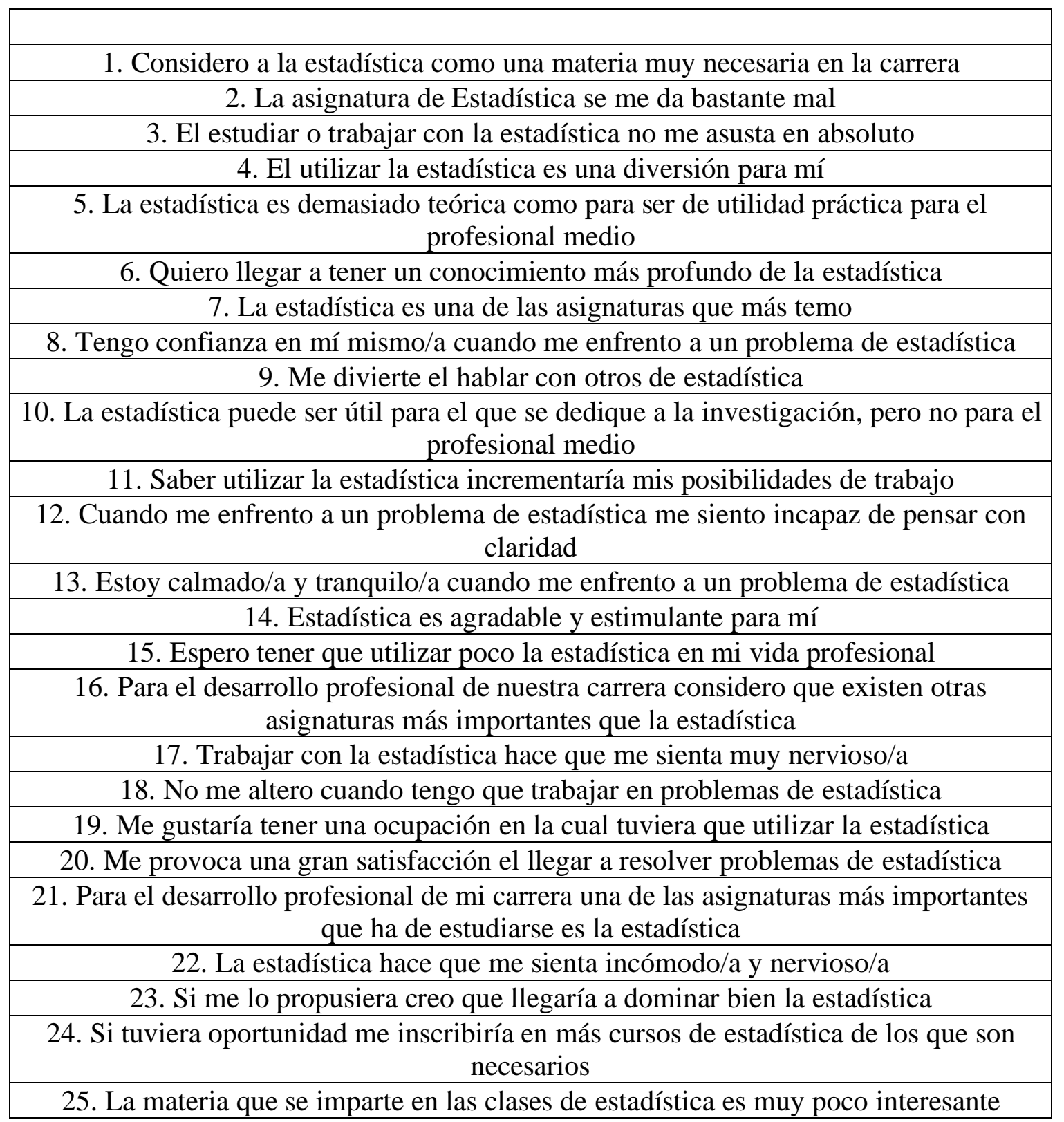

Fuente: Elaboración propia

\section{Instrumento cualitativo}

Para la obtención de datos cualitativos se utilizó el método de la encuesta semiestructurada con preguntas abiertas (segunda encuesta). Esta consistió en un listado de 9 interrogantes para evidenciar conocimientos previos al tomar el curso de estadística sobre: utilidad, habilidades y actitudes hacia la estadística; de este modo se procuró recabar más datos para generar una mejor interpretación.

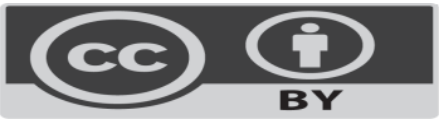




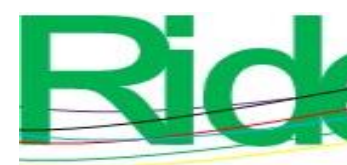

Revista Iberoamericana para la Investigación y el Desarrollo Educativo ISSN 2007 - 7467

\section{Preguntas de la encuesta semiestructurada}

1. ¿Para ti qué es la estadística? ¿Por qué?

2. ¿Alguna vez has tomado la asignatura de estadística? ¿Cómo te ha parecido? ¿Te ha gustado? ¿Por qué?

3. ¿La estadística te parece muy complicada o se te facilita? ¿Por qué?

4. ¿Crees que este curso de estadística te será útil para tu profesión o será una pérdida de tiempo? ¿Por qué?

5. ¿Qué habilidades te gustaría adquirir con esta unidad de aprendizaje?

6. ¿Cuáles son tus expectativas de este curso con respecto al docente?

7. ¿Tienes algunas dificultades para realizar las actividades que te proponen los docentes en las clases? ¿Cuáles son? ¿Por qué?

8. Hasta ahora, ¿cuáles son las mayores lecciones que te ha aportado para la vida tu educación académica?

9. ¿Cuáles tres cosas puedes hacer para mejorar tu aprendizaje este año?

\section{Participantes}

Se llevó a cabo un muestreo no probabilístico por conveniencia e intencional (Lind, Marchal y Mason, 2004; Izcara, 2014; Otzen y Manterola, 2017) a los estudiantes de tercer semestre que cursaban la unidad de aprendizaje de Estadística. La población fue de 76 alumnos (grupo 301, 43 alumnos y grupo 302, 33 alumnos) con edades comprendidas entre los 19 y 22 años de la Escuela Superior de Ciencias Ambientales de la Universidad Autónoma de Guerrero, campus Llano Largo, Acapulco, Guerrero. Vale resaltar que los participantes fueron dos grupos 301 y 302 de los cuales solo contestaron 56 estudiantes, el instrumento cuantitativo conformado por 25 ítems de la encuesta de Auzmendi, antes de tomar el curso de estadística. La segunda fase de la aplicación del mismo instrumento, solo 42 alumnos la contestaron (después de llevar el curso). La encuesta semiestructurada de 9 preguntas abiertas solo la contestaron los 56 alumnos, la cual fue un diagnóstico previo al curso de estadística.

La tercera encuesta la contestaron 51 estudiantes, para evidenciar aspectos cognitivos y la cuarta encuesta sobre la percepción del uso de la estrategia pedagógica del ABP, la contestaron 52 estudiantes. 


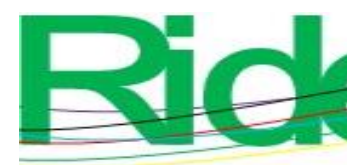

Revista lberoamericana para la Investigación y el Desarrollo Educativo ISSN 2007 - 7467

\section{Métodos de análisis de los datos}

Para el análisis de los datos cuantitativos se utilizaron el paquete estadístico SPSS (versión 21.0) y Excel, los cuales sirvieron para generar tablas y gráficos descriptivos según los criterios de análisis establecidos. La información cualitativa obtenida con las preguntas abiertas se analizó mediante el método de codificación (Rincón, 2014).

\section{Descripción de la implementación de la estrategia pedagógica}

La implementación de la estrategia pedagógica se efectuó del 27 de agosto 2018 al 15 de julio de 2019 durante el horario escolar en los grupos de 301 y 302. Los estudiantes trabajaron con la estrategia pedagógica, para lo cual cada equipo eligió un tema para trabajar en clase; de este tema, luego realizaron un proyecto práctico.

En el diseño del proyecto y en la implementación de la estrategia pedagógica se plantearon las siguientes fases, según el esquema de desarrollo de proyecto de Batanero y Díaz (2011) que se describe a continuación:

\section{Planteamiento de un problema práctico}

Los estudiantes plantearon una problemática práctica de su entorno cotidiano (Batanero y Díaz, 2011; García y Guerrero, 2011; Jonassen, 1997). En esta fase el estudiante eligió el contenido de su proyecto de entre los diferentes temas que se trabajaron en la unidad de aprendizaje Estadística.

\section{Planteamiento de las preguntas de investigación}

Los estudiantes identificaron un problema y posteriormente propusieron soluciones desde diversas perspectivas (Flores y Juárez, 2017). Paralelamente a estas actividades se trabajó en clase, donde se les guió y se aplicaron conceptos, técnicas de los temas de estadística descriptiva, regresión lineal, tamaño de muestra y contraste de hipótesis para que, a través de la estrategia pedagógica del aprendizaje basado en proyectos (ABP), resolvieran el problema. 

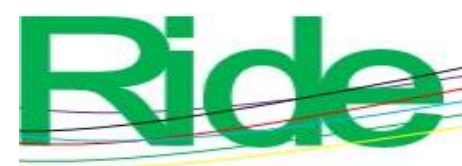

Revista Iberoamericana para la Investigación y el Desarrollo Educativo

ISSN 2007 - 7467

\section{Desarrollo y recolección de datos}

Esta fue la fase de elaboración del proyecto. En primer lugar, los alumnos buscaron información en la biblioteca, revistas e internet para profundizar en el tema elegido. Posteriormente diseñaron el proyecto, siguiendo el guion previamente explicado. Luego se pasó a la justificación del tema y al análisis de necesidades; después se pasó a la descripción de la población, el objetivo de la intervención, los contenidos que se trabajaron en las diferentes actividades, la metodología de trabajo, la calendarización y por último la evaluación.

Asimismo, cada grupo organizó las tareas en función de sus componentes y objetivos planteados, para lo cual se establecieron las condiciones que se debían cumplir en la solución propuesta: se utilizaron métodos y técnicas estadísticas estudiadas en clase para la recogida de datos. El estudiante aplicó los conocimientos revisados en clase.

\section{Organizar, analizar e interpretar los datos}

En esta etapa, los estudiantes desarrollaron un informe de su propuesta de solución aplicando los conocimientos adquiridos; para ello, emplearon métodos y técnicas de organización e interpretación de resultados.

\section{Resuelve el problema y evalúa}

Los estudiantes expusieron su proyecto ante el grupo. Al terminar esta actividad se realizó un análisis que les permitió reflexionar sobre los aprendizajes adquiridos. En este apartado, el reporte escrito final del proyecto siguió la siguiente estructura: título, autores, resumen, introducción, objetivos, materiales, métodos, resultados, discusión y conclusiones, así como referencias, tablas, gráficos y figuras. 


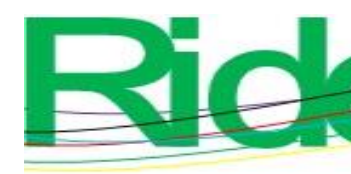

Revista Iberoamericana para la Investigación y el Desarrollo Educativo ISSN 2007 - 7467

\section{Resultados}

\section{Diagnóstico de percepción de actitudes según factores de Auzmendi}

La población estudiada estuvo compuesta por 76 estudiantes (dos grupos), sin embargo solo 56 alumnos contestaron la encuesta en la primera aplicación y 42 en la segunda fase de aplicación de la encuesta de Auzmendi, en la Escuela Superior de Ciencias Ambientales de la UAGro.

La investigación se enfocó en conocer la percepción de los participantes sobre la utilidad, ansiedad, confianza, agrado y motivación de los estudiantes hacia la estadística antes y después de haber desarrollado con ellos una estrategia pedagógica sustentada en el aprendizaje basado en proyectos (ABP).

\section{Factor utilidad}

Este factor se enfocó en medir la productividad o beneficios que puede ofrecer a los estudiantes la estadística para su desarrollo como profesionales (afirmaciones 1, 6, 11, 16, 20 y 21). En tal sentido, se puede decir que en cuanto al ítem 1 (Considero a la estadística como una materia muy necesaria en la carrera), antes de aplicar la estrategia pedagógica $57.1 \%$ (32) y $28.6 \%$ (16) de los alumnos tenía una percepción positiva (totalmente de acuerdo y de acuerdo) hacia la estadística, mientras que luego de aplicar la estrategia pedagógica los porcentajes disminuyeron a $64.3 \%$ (27) y $11.9 \%$ (5) (figura 1). Esta disminución podría explicarse, porque los alumnos piensan que es una materia poco necesaria e importante para la carrera, otros, no había llevado ningún curso de estadística y esto hace que se les complique.

En el ítem 6 (Quiero llegar a tener un conocimiento más profundo de la estadística) se evaluaron los conocimientos y la utilidad hacia la estadística como instrumento para otras áreas del conocimiento. Al respecto, antes de aplicar el curso $58.92 \%$ (33) y $26.78 \%$ (15) de los alumnos estaba totalmente de acuerdo y de acuerdo con el enunciado planteado, mientras que luego de desarrollar el curso los porcentajes se ubicaron en 40.47 \% (17) y $28.57 \%$ (12), respectivamente. Pone en manifiesto el alumno piensa que no es de utilidad la estadística para su formación, esto también se comprueba en el apartado implementación de la estrategia pedagógica. 

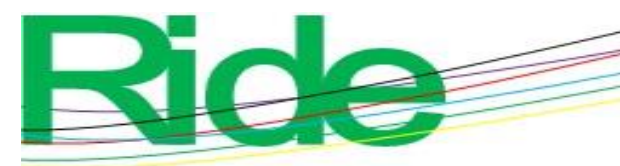

\section{Revista Iberoamericana para la Investigación y el Desarrollo Educativo ISSN 2007 - 7467}

El ítem 11 (Saber utilizar la estadística incrementaría mis posibilidades de trabajo) se enfocó en el sentido positivo de la actitud hacia la estadística. Antes de tomar el curso, $39.28 \%$ (22) y $32.14 \%$ (18) de los alumnos estaba totalmente de acuerdo y de acuerdo con el enunciado 11, mientras que luego del curso aplicado los porcentajes fueron $54.76 \%$ (23) y $16.66 \%(7)$, respectivamente.

El ítem 16 (Para el desarrollo profesional de nuestra carrera considero que existen otras asignaturas más importantes que la estadística) se enfocó en la necesidad de utilizar esta unidad de aprendizaje en la etapa formación educativa. El resultado muestra que antes del curso solo $10.7 \%$ (6) y $35.7 \%$ (20) de los estudiantes estaba totalmente de acuerdo y de acuerdo con el enunciado, mientras que después del curso $16.66 \%$ (7) y $11.90 \%$ (5) opinó de esa forma.

En el ítem 20 (Me provoca una gran satisfacción el llegar a resolver problemas de estadística) antes del curso $21.42 \%$ (12) y $28.57 \%$ (16) de los estudiantes estaba totalmente de acuerdo y de acuerdo con el ítem, mientras que después del curso $30.95 \%$ (13) y 23.80 \% (10) opinó de ese modo.

En la pregunta 21 (Para el desarrollo profesional de mi carrera una de las asignaturas más importantes que ha de estudiarse es la estadística), antes del curso $10.7 \%$ (6) y $35.7 \%$ (20) de los estudiantes estaba totalmente de acuerdo y de acuerdo con el ítem, mientras que después del curso 14.28 \% (6) y $33.33 \%$ (14) opinó de ese modo (figura 1). 


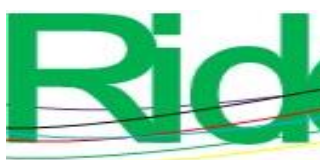

\section{Revista Iberoamericana para la Investigación y el Desarrollo Educativo ISSN 2007 - 7467}

Figura 1. Frecuencias de respuestas de los ítems 1, 6, 11, 16, 20 y 21
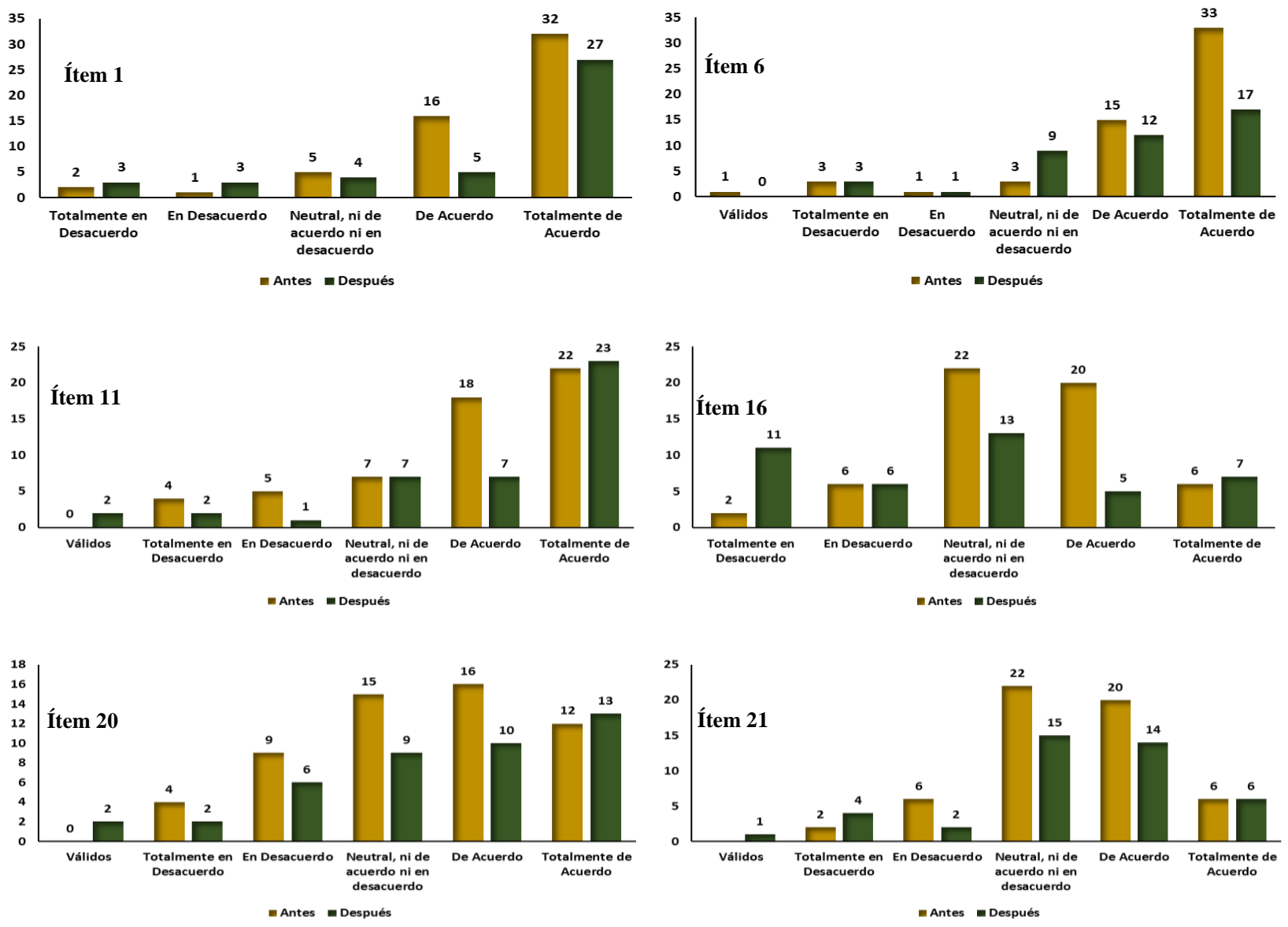

Fuente: Elaboración propia

\section{Factor ansiedad}

Este factor se refiere al temor que el estudiante manifiesta ante la unidad de aprendizaje de Estadística, el cual quedó integrado por los ítems 2, 7, 12, 17 y 22.

En el ítem 2 (La asignatura de Estadística se me da bastante mal) hace alusión al comportamiento del estudiante en el trabajo de clase. En este caso, la pregunta fue formulada de manera inversa. Antes del curso $8.92 \%$ (5) y $17.85 \%$ (10) de los estudiantes estaba totalmente de acuerdo y de acuerdo con el ítem, mientras que después del curso $9.52 \%$ (4) y $16.66 \%$ (7) opinó de ese modo.

En el ítem 7 (La estadística es una de las asignaturas que más temo) antes del curso $7.14 \%$ (4) y $10.7 \%$ (6) de los estudiantes estaba totalmente de acuerdo y de acuerdo con lo planteado en el ítem, mientras que después del curso $14.28 \%$ (6) y 7.14 \% (3) opinó de ese modo. 


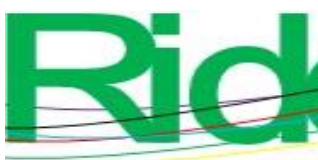

\section{Revista Iberoamericana para la Investigación y el Desarrollo Educativo ISSN 2007 - 7467}

La pregunta 12 (Cuando me enfrento a un problema de estadística me siento incapaz. de pensar con claridad) se enfocó en el temor, el miedo y el componente social de esta unidad de aprendizaje a través de un enunciado negativo. Los resultados demuestran que antes del curso $16.07 \%$ (9) y $16.07 \%$ (9) de los estudiantes están en desacuerdo y totalmente en desacuerdo con lo indicado en el ítem 12, mientras que luego del curso $26.19 \%$ (11) y 16.66 \% (7) opinó así.

En lo concerniente al ítem 17 (Trabajar con la estadística hace que me sienta muy nervioso/a), antes del curso $23.21 \%$ (13) y $17.85 \%$ (10) de los alumnos están en desacuerdo y totalmente en desacuerdo con lo indicado, mientras que después del curso los porcentajes se situaron en 14.28 (6) y $23.80 \%$ (10), respectivamente.

En la pregunta 22 (La estadística hace que me sienta incómodo/a y nervioso/a), antes del curso $16.07 \%$ (9) y $17.85 \%$ (10) de los alumnos están en desacuerdo y totalmente en desacuerdo con lo indicado, mientras que después del curso los porcentajes se situaron en $14.28 \%$ (6) y $28.57 \%$ (12), respectivamente (figura 2).

Figura 2. Frecuencias de respuestas en los ítems 2, 7, 12, 17 y 22
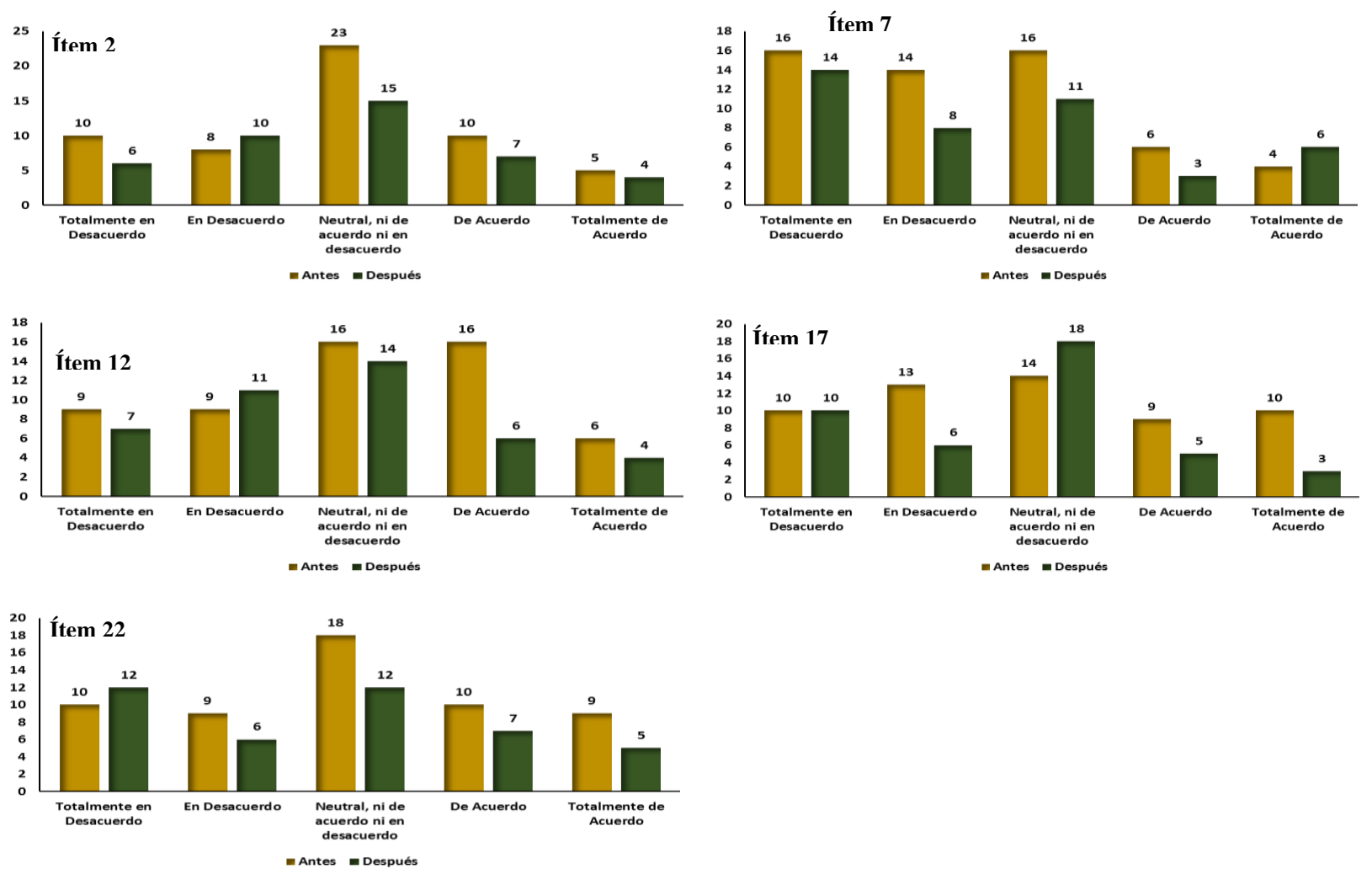

Fuente: Elaboración propia 

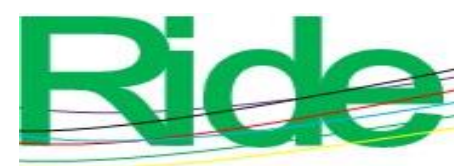

Revista Iberoamericana para la Investigación y el Desarrollo Educativo ISSN 2007 - 7467

\section{Factor confianza}

Este factor se refiere a la seguridad del estudiante en torno a los contenidos de la estadística, y quedó constituido por los ítems 3, 8, 13, 18 y 23.

En la pregunta 3 (El estudiar o trabajar con la estadística no me asusta en absoluto), los resultados indican que antes de desarrollar el curso $30.35 \%$ (17) y $30.35 \%$ (17) de los alumnos estaba totalmente de acuerdo y de acuerdo con lo planteado en el ítem, mientras que después del curso los porcentajes disminuyeron a $11.90 \%$ (5) y $14.28 \%$ (6), respectivamente.

En el ítem 8 (Tengo confianza en mí mismo/a cuando me enfrento a un problema de estadística), antes del curso $23.21 \%$ (13) y $37.5 \%$ (21) de los alumnos estaba totalmente de acuerdo y de acuerdo con lo planteado, mientras que después del curso los porcentajes volvieron a disminuir a $28.57 \%(12)$ y $19.04 \%(8)$, respectivamente.

En la pregunta 13 (Estoy calmado/a y tranquilo/a cuando me enfrento a un problema de estadística), antes del curso $19.64 \%$ (11) y $23.21 \%$ (13) de los estudiantes estaba totalmente de acuerdo y de acuerdo con esa idea, mientras que luego del curso los porcentajes se situaron en $16.66 \%$ (7) y $19.04 \%$ (8), respectivamente.

En cuanto al ítem 18 (No me altero cuando tengo que trabajar en problemas de estadística), antes del curso desarrollado $14.28 \%$ (8) y $32.14 \%$ (18) de los estudiantes estaba totalmente de acuerdo y de acuerdo con el planteamiento, mientras que después del curso $16.66 \%$ (7) y $23.80 \%$ (10) opinaron de ese modo, respectivamente.

Finalmente, en la pregunta 23 (Si me lo propusiera creo que llegaría a dominar bien la estadística) se evidencia que $58.92 \%$ (33) y $23.21 \%$ (13) de los estudiantes estaba totalmente de acuerdo y de acuerdo con el planteamiento, mientras que después del curso $50.00 \%$ (21) y $19.04 \%$ (8) opinaron de ese modo, respectivamente (figura 3). 


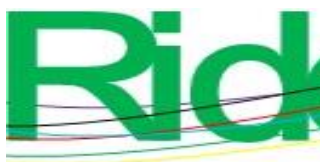

\section{Revista Iberoamericana para la Investigación y el Desarrollo Educativo ISSN 2007 - 7467}

Figura 3. Frecuencias de respuestas en los ítems 3, 8, 13, 18 y 23
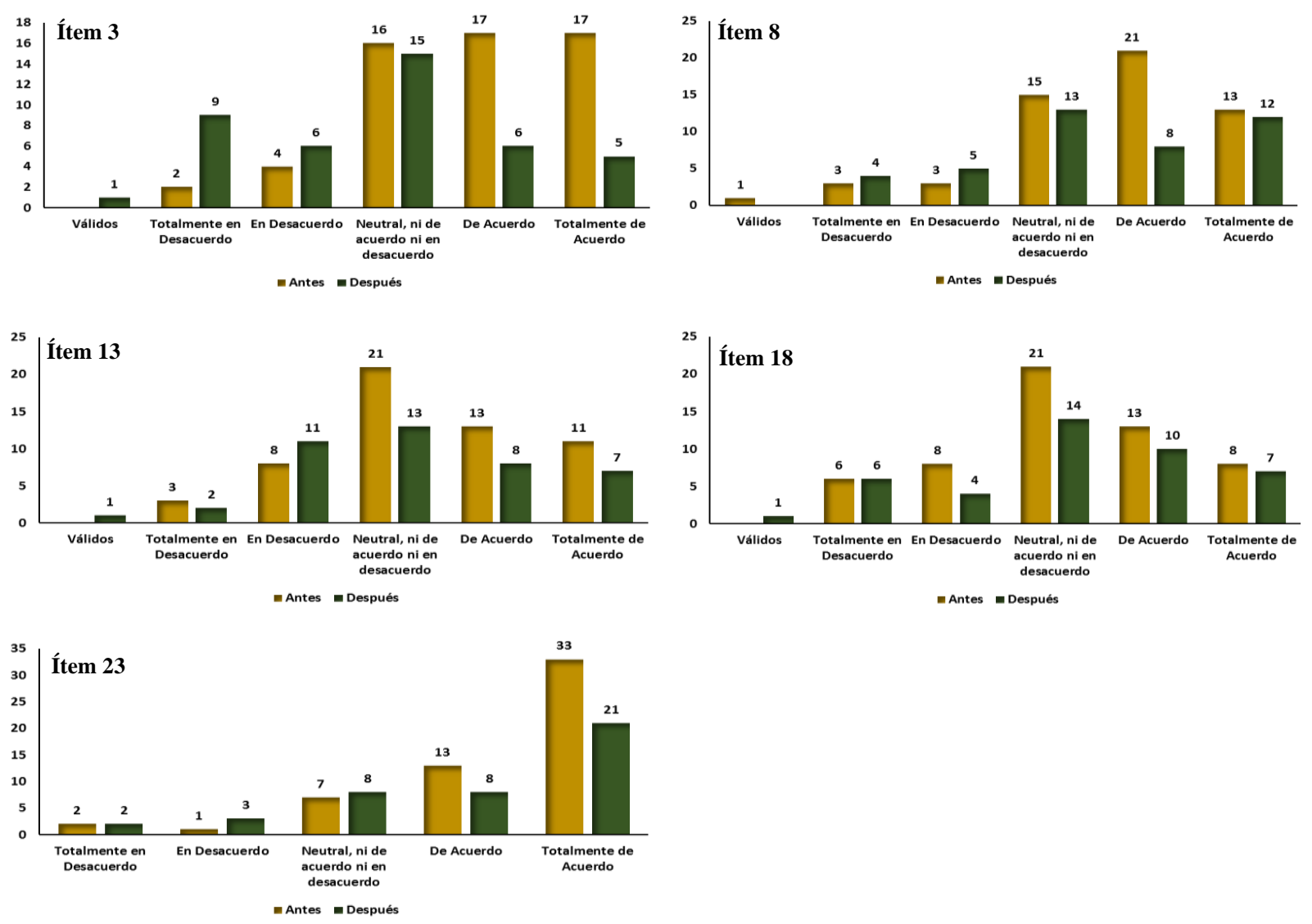

Fuente: Elaboración propia

\section{Factor agrado}

Este factor quedó conformado por los ítems 4, 9, 14, 19 y 24. En la pregunta 4 ( $E l$ utilizar la estadística es una diversión para mí) predominó una postura neutral antes y después de aplicar el curso — $41.07 \%$ (23) y $28.57 \%$ (12), respectivamente_- La causa de esto podría ser que los estudiantes no habían cursado ninguna asignatura de estadística en el nivel medio superior.

Algo similar sucedió con la pregunta 9 (Me divierte el hablar con otros de estadística), pues antes y después de desarrollar el curso predominó una postura neutral en los alumnos: $33.92 \%$ (19) y $38.09 \%$ (16), respectivamente.

En cambio, en la pregunta 14 (Estadística es agradable y estimulante para mî) los alumnos sí se inclinaron por una posición, pues antes del curso 16.07 \% (9) y 33.92 \% (19) 


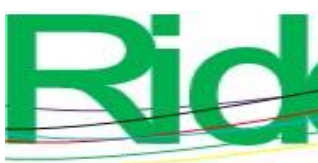

\section{Revista Iberoamericana para la Investigación y el Desarrollo Educativo ISSN 2007 - 7467}

de ellos estuvieron totalmente de acuerdo y de acuerdo con el planteamiento, mientras que después del curso $16.66 \%$ (7) y $28.57 \%$ (12) opinaron de ese modo, respectivamente.

En la pregunta 19 (Me gustaría tener una ocupación en la cual tuviera que utilizar la estadística) también sobresalió una postura neutral, pues antes del curso $48.2 \%$ (27) de los alumnos se pronunciaron de ese modo, mientras que luego del curso ese porcentaje se situó en $30.95 \%$ (13).

Por último, en la pregunta 24 (Si tuviera oportunidad me inscribiría en más cursos de estadística de los que son necesarios), antes del curso $23.21 \%$ (13) y $44.64 \%$ (25) de los estudiantes estaba totalmente de acuerdo y de acuerdo con el planteamiento, mientras que después del curso $26.19 \%$ (11) y $30.95 \%$ (13) opinaron de ese modo, respectivamente (figura 4).

Figura 4. Frecuencias de respuestas en los ítems 4, 9, 14, 19 y 24
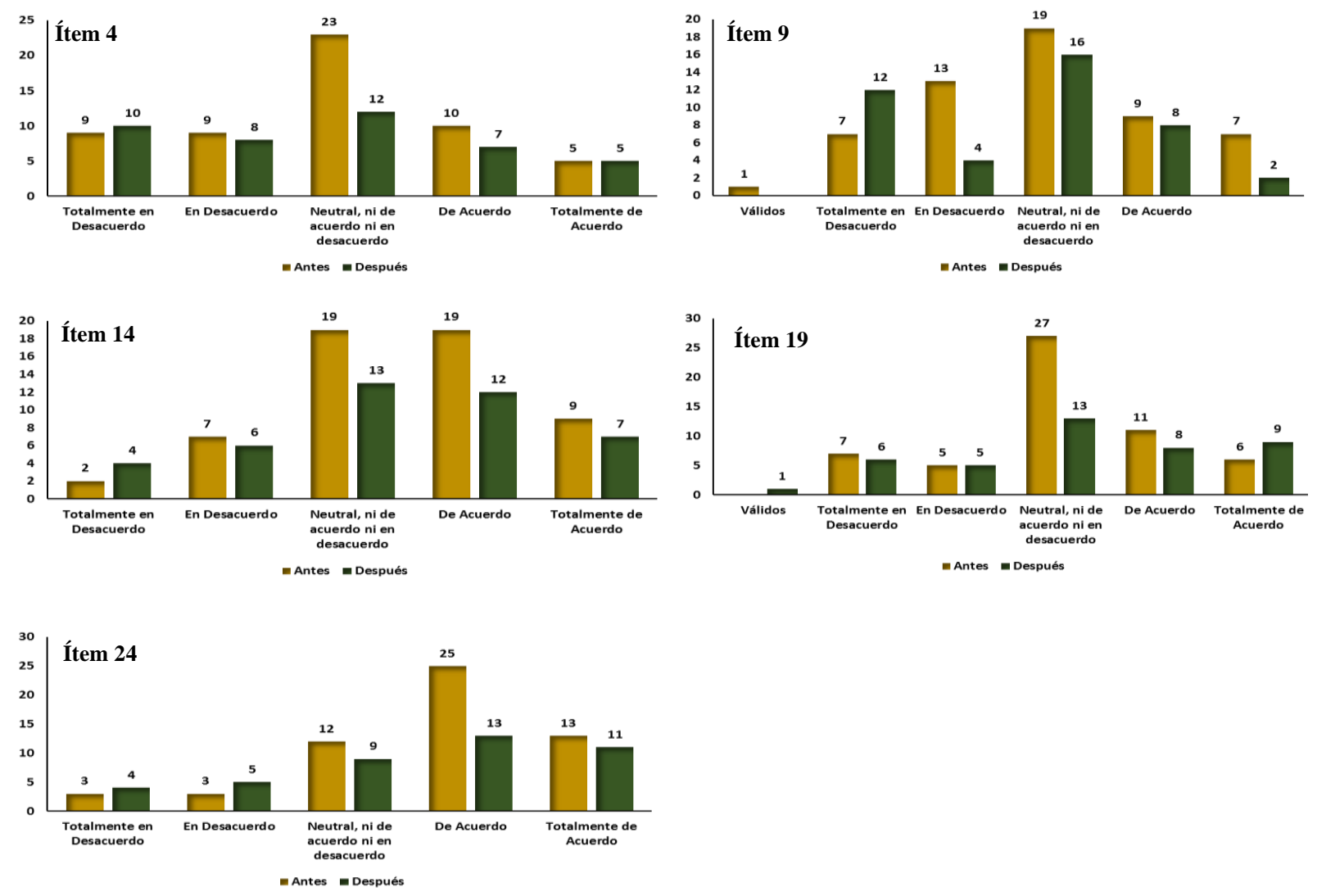

Fuente: Elaboración propia 

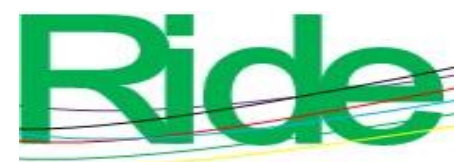

Revista Iberoamericana para la Investigación y el Desarrollo Educativo ISSN 2007 - 7467

\section{Factor motivación}

El factor motivación estuvo conformado por los ítems 5, 10, 15 y 25, los cuales se describen a continuación. En cuanto al ítem 5 (La estadística es demasiado teórica como para ser de utilidad práctica para el profesional medio), antes de curso 28.57 \% (16) y 19.64 $\%$ (11) de los alumnos se mostró en desacuerdo y totalmente en desacuerdo con esa afirmación, mientras que después del curso los porcentajes se ubicaron en $26.19 \%$ (11) y $30.95 \%$ (13), respectivamente.

En la afirmación 10 (La estadística puede ser útil para el que se dedique a la investigación, pero no para el profesional medio), antes del curso $25 \%$ (14) y $16.07 \%$ (9) de los alumnos opinó que se hallaba en desacuerdo y totalmente en desacuerdo con esa idea, mientras que después del curso los porcentajes se ubicaron en $11.90 \%$ (5) y $30.95 \%$ (13), respectivamente.

En cuanto al enunciado 15 (Espero tener que utilizar poco la estadística en mi vida profesional), antes del curso la mayoría de estudiantes asumió una postura neutral —35.7\% (20)—, mientras que luego del curso este porcentaje se situó en 38.09 \% (16).

Finalmente, en la pregunta 25 (La materia que se imparte en las clases de estadística es muy poco interesante), antes del curso $32.14 \%$ (18) y $33.92 \%$ (19) de los alumnos estuvo en desacuerdo y totalmente en desacuerdo con el enunciado, mientras que después del curso los porcentajes se ubicaron en $16.66 \%$ (7) y $35.71 \%$ (15), respectivamente (figura 5). 


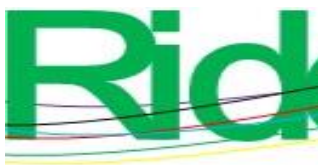

Revista Iberoamericana para la Investigación y el Desarrollo Educativo ISSN 2007 - 7467

Figura 5. Frecuencias de respuestas en los ítems 5, 10, 15 y 25
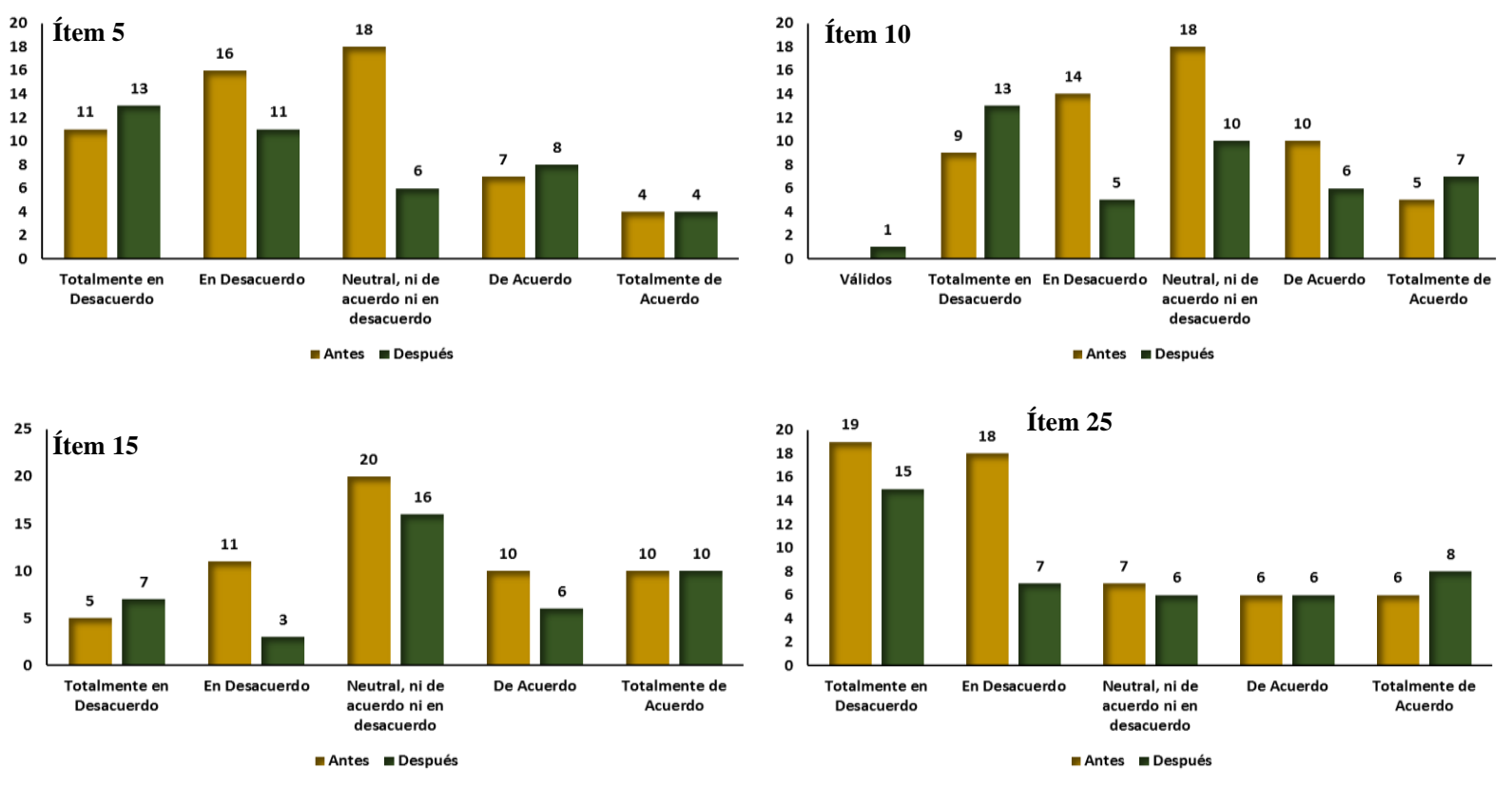

Fuente: Elaboración propia

\section{Implementación de la estrategia pedagógica}

En la tabla 2 se presentan los resultados generales en cuanto a las medias de las puntuaciones obtenidas con la aplicación de la encuesta de actitudes hacia la estadística (conformada por los 25 ítems mencionados).

Sin embargo, antes de enseñar estos resultados, vale acotar que los ítems redactados de forma negativa (p. ej., el número 2: La asignatura de Estadística se me da bastante mal) fueron puntuados en forma inversa para calcular su media. De este modo, una media alta siempre indicará una actitud positiva hacia la estadística.

Ahora bien, los valores medios mejores valorados antes de tomar el curso de estadística fueron los siguientes: considero la estadística como una materia muy necesaria en la carrera, si me lo propusiera creo que llegaría a dominar bien la Estadística (ítems 1 y 23) y quiero llegar a tener un conocimiento más profundo de la estadística (ítem 6) (de los factores utilidad y confianza). En cambio, los peores valorados fueron los aspectos relacionados con la estadística es una de las asignaturas que más temo y la materia que se imparte en las clases de estadística es muy poco interesante (ítems 7 y 25) (de los factores ansiedad y confianza) (tabla 2). 


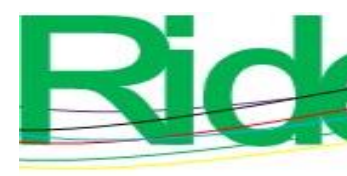

Revista Iberoamericana para la Investigación y el Desarrollo Educativo

ISSN 2007 - 7467

Por otra parte, los valores medios mejores valorados después de tomar el curso de estadística fueron los siguientes: considero a la estadística como una materia muy necesaria en la carrera y si me lo propusiera creo que llegaría a dominar bien la estadística (ítems 1 y 23) (de los factores utilidad y confianza). Los peores corresponden a aspectos relacionados con la estadística es demasiado teórica como para ser de utilidad práctica para el medio profesional y la estadística es una de las asignaturas que más temo (ítems 5 y 7) (de los factores motivación y ansiedad) (tabla 2).

Siguiendo con este análisis de las actitudes negativas y positivas, en la tabla 2 se enseña que antes del curso en las preguntas 2, 4, 5. 7, 9, 10, 16, 17, 22, 25 y después del curso en las preguntas $2,3,4,5.7,9,10,12,16,17,22$ y 25 se evidencian actitudes negativas, pues presentan valores menores a 3 . No obstante, también vale acotar que en los referidos ítems de algunos alumnos se manifestaron de forma positiva, lo cual se demuestra en los siguientes argumentos, estos argumentos fueron recabados con la encuesta semiestructurada con las 9 preguntas abiertas: la estadística como una materia muy necesaria en la carrera, "se me facilita porque es interesante", "llevando la secuencia es sencilla, aunque a veces no entiendo el procedimiento y me pierdo", "bueno, en lo particular trato de entender y comprender la estadística", "sí se me facilita para poder resolver algunas gráficas o datos que se quieren obtener", "no se me complica, pero tampoco se me hace muy fácil".

El estudiar o trabajar con la estadística no me asusta en absoluto: "me parece fácil porque todo se ha visto y sí he entendido", "para los gráficos se me hace fácil hacerlas, los problemas se me complican un poco", "es fácil porque me parece interesante al relacionarlo con todos los datos", "se me facilita, pues son procedimientos sencillos", "me parece una materia sencilla, a veces un poco tediosa".

Quiero llegar a tener un conocimiento más profundo de la estadística: "leer un poco más, ser más responsable y poner más atención en clases”, “estudiar, no quedarme con dudas y poner en práctica lo aprendido", “documentarme más, involucrarme en las diversas actividades escolares y cumplir con tareas y proyectos", "poner más atención de las clases, ser más responsable y estudiar”.

Saber utilizar la estadística incrementaría mis posibilidades de trabajo: “al menos para mí aumentará mis posibilidades de conseguir un trabajo", "si me será útil para cuando ya tenga un trabajo de acuerdo a mi carrera", "es útil saber estadística para mi profesión, ya que se utiliza a diario en cualquier trabajo", "saber estadística es útil en mi profesión". 


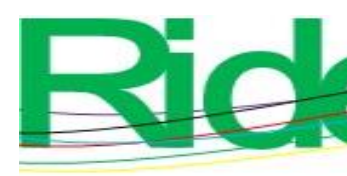

Revista Iberoamericana para la Investigación y el Desarrollo Educativo ISSN 2007 - 7467

Si me lo propusiera creo que llegaría a dominar bien la estadística: "es una meta de la vida tener conocimiento estadístico y para ello es necesario disciplinarme, aplicarme y tener más responsabilidad", "ser más responsable cada día y a ponerle más interés a mi aprendizaje hacia la estadística", "estudiar, no quedarme con dudas y poner en práctica lo aprendido".

Como se puede apreciar en estos fragmentos, las explicaciones anteriores están vinculadas a los factores utilidad y confianza, pues de forma general se refieren a la utilidad de ese conocimiento para su futuro profesional; esto se realizó con base en el análisis de los resultados del cuestionario cuantitativo, encuesta de Auzmendi y cualitativos con base a la encuesta semiestructurada de 9 preguntas abiertas.

Por otra parte, de los argumentos negativos destacamos los siguientes: la asignatura de Estadística se me da bastante mal: "sí, nunca había tomado el curso hasta ahorita, y sí me está gustando porque aprendes a graficar muy bien”, "es la primera vez que la tomo, sí me gusta aprender esta materia, que la lleve en bachilleres”.

La materia que se imparte en las clases de estadística es muy poco interesante: "es poco interesante las formas de hacerlo, me parece un poco complicada, pero útil, me agrada un poco y posiblemente necesaria”, “en la preparatoria llevé el curso de estadística, me pareció muy interesante, ya que la profesora nos explicaba muy bien, me gustó porque era muy interesante".

En cuanto a La estadística es demasiado teórica como para ser de utilidad práctica para el profesional medio y La Estadística puede ser útil para el que se dedique a la investigación, pero no para el profesional medio, se ofrecen las siguientes opiniones: “al menos para mí será un poco útil, porque por ejemplo en cualquier trabajo nos pueden poner a graficar y creo para cualquier trabajo se utilizara alguna vez", "no es teórica y sí me será útil para cuando ya tenga una carrera, para cuando ya tenga un trabajo de acuerdo a mi carrera", "creo que si está en el plan de estudio es por algo, yo creo que sí es muy buena en la carrera".

En resumen, se puede observar que estos testimonios están vinculados al componente cognitivo (utilidad, ansiedad, agrado y motivación). Asimismo, se puede apreciar que algunos alumnos no han llevado un curso de estadística durante su formación en el nivel medio superior, mientras que quienes sí han cursado este tipo de asignaturas pareciera que han olvidado ese contenido debido a que en el momento les pareció poco útil o no fueron motivados de forma adecuada sobre los beneficios de ese conocimiento para la vida 


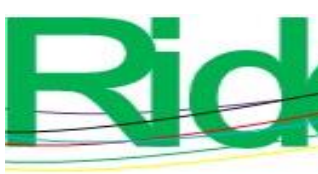

Revista Iberoamericana para la Investigación y el Desarrollo Educativo

ISSN 2007 - 7467

cotidiana. Por ello, se puede decir que hace falta crear estrategias que motiven más y que muestren la utilidad de la asignatura.

Tabla 2. Medias de las puntuaciones obtenidas con la aplicación de la encuesta de actitudes hacia la estadística (antes y después de tomar el curso)

\begin{tabular}{|c|c|c|}
\hline & Antes & Después \\
\hline & Media & Media \\
\hline $\begin{array}{l}\text { 1. Considero a la estadística como una materia muy necesaria en la } \\
\text { carrera }\end{array}$ & 4.34 & 4.19 \\
\hline 2. La asignatura de Estadística se me da bastante mal & 2.86 & 2.83 \\
\hline 3. El estudiar o trabajar con la estadística no me asusta en absoluto & 3.77 & 2.74 \\
\hline 4. El utilizar la estadística es una diversión para mí & 2.88 & 2.74 \\
\hline $\begin{array}{l}\text { 5. La estadística es demasiado teórica como para ser de utilidad } \\
\text { práctica para el profesional medio }\end{array}$ & 2.59 & 2.5 \\
\hline 6. Quiero llegar a tener un conocimiento más profundo de la estadística & 4.27 & 3.93 \\
\hline 7. La estadística es una de las asignaturas que más temo & 2.43 & 2.5 \\
\hline $\begin{array}{l}\text { 8. Tengo confianza en mí mismo/a cuando me enfrento a un problema } \\
\text { de estadística }\end{array}$ & 3.63 & 3.45 \\
\hline 9. Me divierte el hablar con otros de estadística & 2.88 & 2.62 \\
\hline $\begin{array}{l}\text { 10. La estadística puede ser útil para el que se dedique a la } \\
\text { investigación, pero no para el profesional medio }\end{array}$ & 2.79 & 2.67 \\
\hline $\begin{array}{l}\text { 11. Saber utilizar la estadística incrementaría mis posibilidades de } \\
\text { trabajo }\end{array}$ & 3.88 & 4 \\
\hline $\begin{array}{l}\text { 12. Cuando me enfrento a un problema de estadística me siento incapaz } \\
\text { de pensar con claridad }\end{array}$ & 3.02 & 2.74 \\
\hline $\begin{array}{l}\text { 13. Estoy calmado/a y tranquilo/a cuando me enfrento a un problema } \\
\text { de estadística }\end{array}$ & 3.38 & 3.1 \\
\hline 14. Estadística es agradable y estimulante para mí & 3.46 & 3.29 \\
\hline 15. Espero tener que utilizar poco la estadística en mi vida profesional & 3.16 & 3.21 \\
\hline $\begin{array}{l}\text { 16. Para el desarrollo profesional de nuestra carrera considero que } \\
\text { existen otras asignaturas más importantes que la estadística }\end{array}$ & 2.88 & 2.79 \\
\hline 17. Trabajar con la estadística hace que me sienta muy nervioso/a & 2.93 & 2.64 \\
\hline 18. No me altero cuando tengo que trabajar en problemas de estadística & 3.16 & 3.12 \\
\hline $\begin{array}{l}\text { 19. Me gustaría tener una ocupación en la cual tuviera que utilizar la } \\
\text { estadística }\end{array}$ & 3.07 & 3.14 \\
\hline $\begin{array}{l}\text { 20. Me provoca una gran satisfacción el llegar a resolver problemas de } \\
\text { estadística }\end{array}$ & 3.41 & 3.48 \\
\hline $\begin{array}{l}\text { 21. Para el desarrollo profesional de mi carrera una de las asignaturas } \\
\text { más importantes que ha de estudiarse es la estadística }\end{array}$ & 3.39 & 3.31 \\
\hline 22. La estadística hace que me sienta incómodo/a y nervioso/a & 2.98 & 2.69 \\
\hline 23. Si me lo propusiera creo que llegaría a dominar bien la estadística & 4.32 & 4.02 \\
\hline $\begin{array}{l}\text { 24. Si tuviera oportunidad me inscribiría en más cursos de estadística } \\
\text { de los que son necesarios }\end{array}$ & 3.75 & 3.52 \\
\hline $\begin{array}{l}\text { 25. La materia que se imparte en las clases de estadística es muy poco } \\
\text { interesante }\end{array}$ & 2.32 & 2.64 \\
\hline
\end{tabular}

Fuente: Elaboración propia 


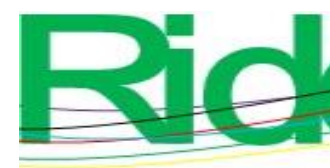

Revista Iberoamericana para la Investigación y el Desarrollo Educativo ISSN 2007 - 7467

\section{Aspectos cognitivos}

La encuesta para medir conocimientos después de llevar el curso de estadística la respondieron solo 51 de estudiantes (dos grupos). En la tabla 3 se recogen los resultados en cuanto a número de aciertos y porcentaje de aciertos en cada una de las preguntas de la encuesta de conocimientos adquiridos de la unidad de aprendizaje de Estadística de la licenciatura en Ciencias Ambientales.

Tabla 3. Resultados de la encuesta para medir conocimiento

\begin{tabular}{|l|c|c|}
\hline \multicolumn{1}{|c|}{ Pregunta } & Aciertos & $\begin{array}{c}\text { Porcentaje de } \\
\text { aciertos }\end{array}$ \\
\hline 1) Tipo de variable estadística. & 31 & $60.8 \%$ \\
\hline 2) Pregunta sobre la mediana. & 46 & $90.2 \%$ \\
\hline 3) Pregunta sobre la media. & 38 & $74.5 \%$ \\
\hline 4) Tipo de gráfico. & 48 & $94.1 \%$ \\
\hline 5) Diagrama de cajas y bigotes. & 8 & $15.7 \%$ \\
\hline 6) Tamaño de la muestra. & 39 & $76.5 \%$ \\
\hline 7) Análisis de regresión lineal & 45 & $88.2 \%$ \\
\hline 8) Contraste de hipótesis & 32 & $62.7 \%$ \\
\hline 9) Modelo lineal simple & 29 & $56.9 \%$ \\
\hline 10) Recta de regresión & 10 & $19.6 \%$ \\
\hline
\end{tabular}

Fuente. Elaboración propia con base en Ayuga, González, Grande y Martínez (2012).

Las preguntas 1, 2, 3, 4, 6, 7, 8 y 9 adquirieron un porcentaje de aciertos superior a $60 \%$. Estas preguntas corresponden a los bloques de estadística descriptiva, tamaño de muestra, análisis de regresión y contraste de hipótesis. En cambio, las dos preguntas restantes (5 y 10) no superaron el $20 \%$ de aciertos.

En la figura 6(a) se muestran las respuestas obtenidas para las cinco primeras preguntas de la encuesta de evaluación, correspondientes al bloque temático estadística descriptiva. Se han marcado en color rojo las respuestas correctas de cada pregunta. Obsérvese que la mayoría de los alumnos contestaron correctamente solo en la pregunta 5, en la que la mayoría optó por la moda, lo que muestra que no se sabe interpretar el gráfico de cajas. Asimismo, la respuesta mayoritaria en la pregunta 1 fue la opción $a$, que representa el tipo de variable discreta, lo que demuestra que conocen los tipos de variables. En el caso de la pregunta 2, los aciertos (inciso c) permiten deducir que los estudiantes dominan más el cálculo de la mediana. Lo mismo sucede en el caso del cálculo de la media (inciso b) y la representación de los gráficos (inciso c), por lo que se puede decir que dominan la estadística descriptiva. 

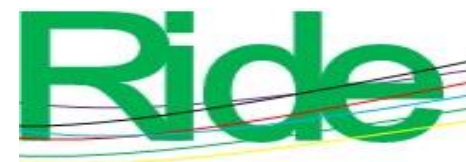

Revista Iberoamericana para la Investigación y el Desarrollo Educativo

ISSN 2007 - 7467

Por otra parte, en la figura 6(b) se muestran las respuestas a las preguntas del bloque temático tamaño de muestra, contraste de hipótesis y regresión lineal (ítems 6 a 10). En estos bloques, de las tres opciones, la respuesta correcta (señalada en rojo) fue escogida de forma mayoritaria en cuatro de las cinco preguntas, salvo en la pregunta 10. En esta pregunta la respuesta más escogida fue el inciso c) que, en la forma de realizar la regresión lineal o modelación lineal, confunde o no tiene claro sobre el tema que trata la pregunta. A partir de este dato, se puede inferir que los estudiantes tienen conocimientos sobre los bloques de tamaño de muestra, contraste de hipótesis y regresión lineal.

Figura 6. Respuestas obtenidas de bloque de estadística descriptiva, bloque tamaño de muestra, contraste de hipótesis y regresión lineal
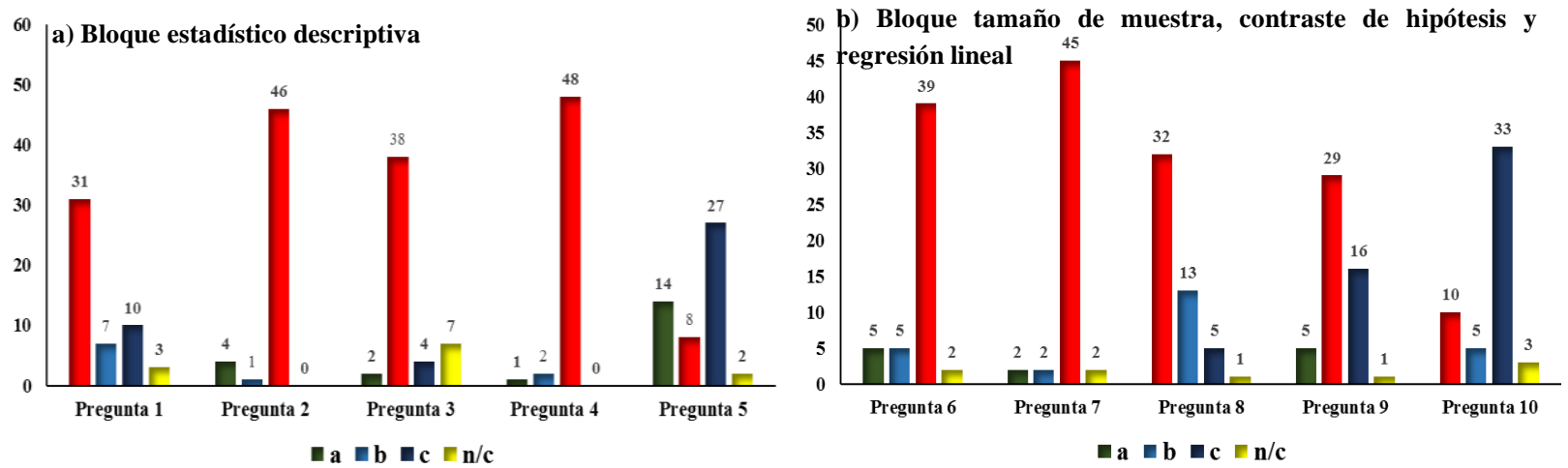

Fuente: Elaboración propia

\section{Estrategia pedagógica con base en el aprendizaje basado en proyecto (ABP)}

Al comenzar el curso, se les planteó a los estudiantes la realización y ejecución de un proyecto, en el cual se aplicarían los conceptos teóricos vistos en las clases de estadística descriptiva, tamaño de muestra, contraste de hipótesis y regresión lineal.

El resultado sobre la percepción del uso de la estrategia pedagógica del ABP se muestra en la tabla 4. Esta encuesta la contestaron solo 52 estudiantes (integrado por dos grupos). En la primera pregunta, la mayoría (94.2\%) de los estudiantes consideraron que la estrategia pedagógica implementada es un apoyo importante en su desarrollo profesional.

En la segunda pregunta, la mitad consideró que fue difícil encontrar una idea de proyecto para desarrollarlo en el curso de estadística. En la pregunta 3, sobre si se 


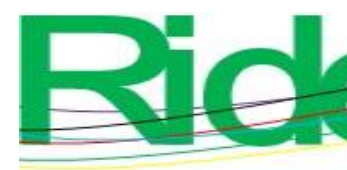

\section{Revista Iberoamericana para la Investigación y el Desarrollo Educativo ISSN 2007 - 7467}

explicaron los temas y conceptos vistos en clase para la solución de los problemas del proyecto, solo $11.5 \%$ de los estudiantes respondieron de forma negativa.

Según los resultados de la pregunta 4, la mayoría (80.8\%) de los estudiantes consideraron que con la estrategia pedagógica se trabajó en forma apropiada durante el semestre. Sobre la pregunta 5, 86.5\% de los estudiantes encuestados estuvieron conforme con la forma de realizar los trabajos. En ese sentido, los estudiantes a medida que avanzaron en el proyecto demostraron una mejoría, mayor capacidad para sintetizar y analizar los resultados que tenían parcialmente de sus avances.

En la pregunta 6 se averiguó la percepción de los estudiantes sobre la valoración del trabajo final (exposición oral y escrita, así como porcentaje total de la evaluación del semestre que corresponde a $40 \%$ ). En este sentido, $63.5 \%$ de los estudiantes encuestados estuvo de acuerdo con dicha valoración.

Sobre la pregunta 7, la mayoría de los estudiantes encuestados (67.3\%) consideraron que el tiempo para el desarrollo de la parte práctica del proyecto fue suficiente; sin embargo, un número de encuestados se manifestó de forma negativa (28.8 $\%$ ), pues argumentaron que el tiempo fijado no fue lo suficiente por los problemas logísticos que tuvieron de sus actividades escolares.

En la pregunta 8, más de la mitad de los estudiantes (69.2\%) manifestaron que la enseñanza a través de la estrategia pedagógica realizada satisfizo sus expectativas de educación, por lo que hicieron diversos comentarios sobre la importancia de este tipo de iniciativas.

En la pregunta 9, casi la mitad $(44.1 \%)$ de los encuestados opinó que las condiciones y falta de equipos dificultó el desarrollo del proyecto. En este sentido, vale indicar que se presentaron inconvenientes de programación y logística con algunos equipos para desarrollar las actividades prácticas de campo, principalmente. Sin embargo, también se puede decir que este tipo de situaciones se pueden usar para desarrollar el ingenio y la imaginación de los alumnos, pues en la vida cotidiana y laboral ese tipo de inconvenientes pueden presentarse.

En la pregunta 10, $76.9 \%$ los encuestado consideraron que se cumplieron las expectativas sobre la gestión realizada por el docente en el desarrollo del proyecto durante el curso de estadística. 
Tabla 4. Resultados de la encuesta sobre la implementación de la estrategia pedagógica en la Escuela Superior de Ciencias Ambientales

\begin{tabular}{|c|c|c|c|c|c|c|}
\hline Pregunta & Sí & $\%$ & \begin{tabular}{l|l}
$\mathbf{N}$ \\
$\mathbf{0}$
\end{tabular} & $\%$ & $\mathbf{N} / \mathrm{C}$ & $\%$ \\
\hline $\begin{array}{l}\text { 1. ¿Cree usted que trabajar con proyecto en clase es } \\
\text { importante para su formación profesional? }\end{array}$ & 49 & $\begin{array}{c}94.2 \\
\%\end{array}$ & 3 & $5.8 \%$ & & $\begin{array}{c}0.00 \\
\%\end{array}$ \\
\hline $\begin{array}{l}\text { 2. ¿Le fue difícil encontrar una idea de proyecto apropiado } \\
\text { para desarrollar en el curso de estadística? }\end{array}$ & 26 & $\begin{array}{c}50.0 \\
\%\end{array}$ & 26 & $\begin{array}{c}50.0 \\
\%\end{array}$ & & $\begin{array}{c}0.00 \\
\%\end{array}$ \\
\hline $\begin{array}{l}\text { 3. ¿Se explicaron y aplicaron los temas y conceptos vistos } \\
\text { en clase para la solución de los problemas del proyecto de } \\
\text { clase? }\end{array}$ & 46 & $\begin{array}{c}88.5 \\
\%\end{array}$ & 6 & $\begin{array}{c}11.5 \\
\%\end{array}$ & & $\begin{array}{c}0.00 \\
\%\end{array}$ \\
\hline $\begin{array}{l}\text { 4. ¿Cree usted que la forma de realizar el proyecto durante } \\
\text { el semestre fue la adecuada? }\end{array}$ & 42 & $\begin{array}{c}80.8 \\
\%\end{array}$ & 10 & $\begin{array}{c}19.2 \\
\%\end{array}$ & & $\begin{array}{c}0.00 \\
\%\end{array}$ \\
\hline $\begin{array}{l}\text { 5. ¿Está conforme con la forma de realizar los trabajos y } \\
\text { (entrega) del proyecto durante el semestre? }\end{array}$ & 45 & $\begin{array}{c}86.5 \\
\%\end{array}$ & 7 & $\begin{array}{c}13.5 \\
\%\end{array}$ & & $\begin{array}{c}0.00 \\
\%\end{array}$ \\
\hline $\begin{array}{l}\text { 6. ¿Considera que la valoración final del proyecto } \\
\text { corresponda al } 40 \% \text { de la calificación final de la unidad } \\
\text { de aprendizaje? }\end{array}$ & 33 & $\begin{array}{c}63.5 \\
\%\end{array}$ & 19 & $\begin{array}{c}36.5 \\
\%\end{array}$ & & $\begin{array}{c}0.00 \\
\%\end{array}$ \\
\hline $\begin{array}{l}\text { 7. ¿El tiempo estipulado para el desarrollo del proyecto es } \\
\text { suficiente? }\end{array}$ & 35 & $\begin{array}{c}67.3 \\
\%\end{array}$ & 15 & $\begin{array}{c}28.8 \\
\%\end{array}$ & 2 & $\begin{array}{c}3.85 \\
\%\end{array}$ \\
\hline $\begin{array}{l}\text { 8. ¿Este tipo de metodologías para el aprendizaje } \\
\text { satisfacen sus expectativas como estudiante si se compara } \\
\text { con otros métodos tradicionales de enseñanza? }\end{array}$ & 36 & $\begin{array}{c}69.2 \\
\%\end{array}$ & 15 & $\begin{array}{c}28.8 \\
\%\end{array}$ & 1 & $\begin{array}{c}1.92 \\
\%\end{array}$ \\
\hline $\begin{array}{l}\text { 9. ¿Considera que los materiales, equipos de laboratorios } \\
\text { y demás recursos disponibles actualmente son los } \\
\text { adecuados para el desarrollo de la parte experimental del } \\
\text { proyecto? }\end{array}$ & 27 & $\begin{array}{c}51.9 \\
\%\end{array}$ & 23 & $\begin{array}{c}44.2 \\
\%\end{array}$ & 2 & $\begin{array}{c}3.85 \\
\%\end{array}$ \\
\hline $\begin{array}{l}\text { 10. ¿Usted considera que la orientación para el desarrollo } \\
\text { del trabajo por parte del docente cumplió con sus } \\
\text { expectativas? }\end{array}$ & 40 & $\begin{array}{c}76.9 \\
\%\end{array}$ & 11 & $\begin{array}{c}21.1 \\
\%\end{array}$ & 1 & $\begin{array}{c}1.92 \\
\%\end{array}$ \\
\hline
\end{tabular}

Nota: Las preguntas de la tabla 4 fueron tomadas y adaptadas de Rodríguez y Cortés (2010)

Fuente: Elaboración propia

\section{Discusión}

Esta investigación se enfocó, en primer lugar, en analizar las actitudes hacia el aprendizaje y los conocimientos elementales de la estadística de estudiantes de la licenciatura en Ciencias Ambientales de la Escuela Superior de Ciencias Ambientales de la Universidad Autónoma de Guerrero. Luego, con base en los resultados recabados, se aplicó una estrategia pedagógica pertinente para orientar el proceso de enseñanza y disminuir el rezago en la referida asignatura. 


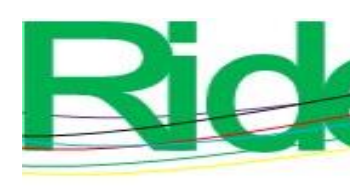

Revista Iberoamericana para la Investigación y el Desarrollo Educativo

ISSN $2007-7467$

En ese sentido, se puede decir que el proceso de la estrategia de aprendizaje basado en proyecto se convierte en una pieza clave para entender la actitud de los estudiantes hacia la estadística y, sobre todo, para procurar modificar la forma en que ellos construyen su aprendizaje. En concreto, y a partir de los resultados obtenidos, una estrategia pedagógica de esta naturaleza podría servir para cambiar significativamente la actitud de los estudiantes y mejorar los resultados académicos en unidades de aprendizajes con contenido estadístico.

Los resultados recabados, por tanto, nos llevan a pensar que la actitud hacia la estadística se basa en el gusto hacia la disciplina. En tal sentido, los alumnos reconocen la importancia y utilidad de esta área disciplinar, lo cual se evidencia en los hallazgos conseguidos en el ítem 1 (Considero a la estadística como una materia muy necesaria en la carrera), ítem 6 (Quiero llegar a tener un conocimiento más profundo de la estadística), ítem 11 (Saber utilizar la estadística incrementaría mis posibilidades de trabajo), ítem 16 (Para el desarrollo profesional de nuestra carrera considero que existen otras asignaturas más importantes que la estadística); ítem 20 (Me provoca una gran satisfacción el llegar a resolver problemas de estadística) e ítem 21 (Para el desarrollo profesional de mi carrera una de las asignaturas más importantes que ha de estudiarse es la estadística). Estos resultados coinciden con los encontrados por Salinas y Mayén (2016), Rojas, Escalera, Moreno y García (2017) y Sánchez (2019).

En cuanto al factor ansiedad (integrado por los ítems 2, 7, 12, 17 y 22), después del curso: se puede indicar que 38.1 \% de los alumnos están en desacuerdo con el ítem 2 ( $L a$ asignatura de Estadística se me da bastante mal); asimismo, 52.4\% no está de acuerdo con el ítem 7 (La estadística es una de las asignaturas que más temo). En el ítem 12 (Cuando me enfrento a un problema de estadística me siento incapaz de pensar con claridad), la mayoría (76.2\%) de la muestra dividió su respuesta de manera neutral y en desacuerdo. En el ítem 17 (Trabajar con la estadística hace que me sienta muy nervioso/a), la mayoría también (81 \%) dividió su respuesta. Estos resultados coinciden con los encontrado por García et al. (2016), Rojas et al. (2017) y Sánchez (2019).

En el factor confianza (preguntas 3, 8, 13, 18 y 23), la mayoría de los estudiantes de haber cursado la unidad de aprendizaje respondió de manera neutral a la pregunta 3 y 8 ( $E l$ estudiar o trabajar con la estadística no me asusta en absoluto y Tengo confianza en mí mismo/a cuando me enfrento a un problema de estadística) (35.7 \% y $31 \%$, respectivamente). Para la pregunta 13 (Estoy calmado/a y tranquilo/a cuando me enfrento a un problema de estadística), $33.3 \%$ de los estudiantes manifestó que se sentía calmado al 


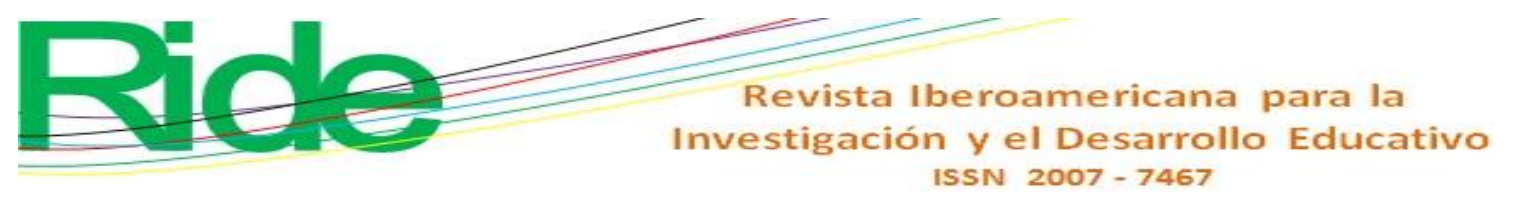

trabajar con un problema estadístico. Asimismo, para la pregunta 18 (No me altero cuando tengo que trabajar en problemas de estadística) la mayoría de los estudiantes está de acuerdo con lo planteado. Estos hallazgos coinciden con la investigación de Sánchez (2019).

En cuanto al factor agrado (ítems 4, 9, 14, 19 y 24), se puede decir que en el ítem 4 (El utilizar la estadística es una diversión para mî) se registró una actitud desfavorable hacia la estadística. En la pregunta 9 (Me divierte el hablar con otros de estadística), la mayoría de los estudiantes contestaron de forma neutral o estaban en desacuerdo con lo sugerido. Sin embargo, en la pregunta 14 (Estadística es agradable y estimulante para mí) se evidenció que los estudiantes tienen una actitud desfavorable hacia la estadística. De igual forma, en la pregunta 19 (Me gustaría tener una ocupación en la cual tuviera que utilizar la estadística) los estudiantes manifestaron que estaban en desacuerdo o eran neutrales frente a lo indicado. En la pregunta 24 (Si tuviera oportunidad me inscribiría en más cursos de estadística de los que son necesarios) los estudiantes se mostraron neutral a la afirmación. Estos son similares a los de Sánchez (2019).

En el factor motivación (integrado por los ítems 5, 10, 15, 20 y 25), se encontró que la mayoría $(57.1 \%$ ) de los estudiantes están en desacuerdo con la afirmación 5 (La estadística es demasiado teórica como para ser de utilidad práctica para el profesional medio). Sin embargo, consideran que es de utilidad para el profesional que no se dedique a la investigación. Estos resultados de igual forma son similares a los encontrados por Sánchez (2019).

De forma general, los hallazgos indican que la mayoría de los 56 estudiantes de la muestra evidenciaron una actitud positiva hacia la estadística, resultados similares a los encontrados por Bautista et al. (2016), quienes trabajaron con alumnos de diferentes carreras de la Universidad de Sonora.

Asimismo, en cuanto a los resultados de la implementación de la estrategia de aprendizaje basada en proyecto, se puede afirmar que la gran aceptación, la actitud positiva y el sentido de utilidad encontrado hacia la estadística por parte de los alumnos coindice con lo reportado por Astorga, Flores, Ibarra, Mariscal y Vizcarra (2017), Inzunza (2017), Flores y Juárez (2017) y Toledo, Arellano, Aguilar y Molina (2018) en nivel de licenciatura de diferentes universidades de México.

Finalmente, podemos decir que esta investigación aporta evidencia para mejorar el proceso de enseñanza y aprendizaje, así como para motivar al estudiante en torno a las 


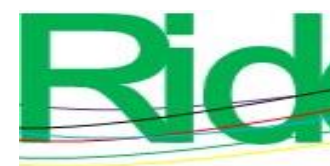

Revista Iberoamericana para la Investigación y el Desarrollo Educativo ISSN 2007 - 7467

ventajas que ofrece la estadística no solo para la vida cotidiana, sino también para el buen desempeño profesional.

\section{Conclusiones}

A partir de las preguntas de la encuesta de Auzmendi, se puede concluir que los estudiantes percibieron la utilidad de la estadística, pues $85.7 \%$ de ellos consideran que esa disciplina es una muy necesaria en la carrera de ciencias ambientales, por lo que quieren tener un conocimiento más profundo de la estadística.

Asimismo, $71.5 \%$ considera que saber utilizar la estadística incrementará sus posibilidades de trabajo, y a la mitad de los participantes les provoca una gran satisfacción cuando resuelven problemas de estadística. En este sentido, $47.6 \%$ de los estudiantes demostraron que eran conscientes de la importancia de la estadística en su formación, aunque también vale comentar que otro grupo de alumnos considera que "para el desarrollo profesional de nuestra carrera considero que existen otras asignaturas más importantes que la estadística” — este ítem fue el único que consiguió una media inferior a 3 (2.88)—.

En cuanto al componte ansiedad, los estudiantes han evidenciado una percepción negativa hacia la estadística por la capacidad que esta pueda tener en tergiversar los resultados de un estudio. De hecho, en el ítem La asignatura de Estadística se me da bastante mal, $26.8 \%$ de los encuestados están de acuerdo con la afirmación. En el ítem La estadística es una de las asignaturas que más temo, más de la mitad se manifestó de forma neutral. Sobre Cuando me enfrento a un problema de estadística me siento incapaz de pensar con claridad, la tercera parte de los encuestados (39.3\%) están de acuerdo con la afirmación, mientras que en Trabajar con la estadística hace que me sienta muy nerviosola y La estadística hace que me sienta incómodo/a y nervioso/a, estos dos ítems consiguieron 39.55 $\%$ en cuanto a desacuerdo con la afirmación. Algunas de las justificaciones de los alumnos al respecto fueron las siguientes: "nunca había tomado el curso hasta ahorita", "es la primera vez que la tomo, que la lleve en bachilleres" y "es la primera vez que llevo esta materia".

En cuanto al confianza, se puede concluir que los estudiantes de Ciencias Ambientales tienen actitudes positivas hacia la estadística como forma de razonamiento, según lo evidencian los ítem 1, 6, 11, 16 y 21, los cuales alcanzaron medias superiores a 3.

También se puede afirmar que los estudiantes ven con agrado a la estadística, e incluso les gustaría tener una ocupación en la cual tuvieran que utilizar ese conocimiento. 


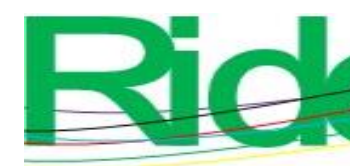

Revista Iberoamericana para la Investigación y el Desarrollo Educativo

ISSN 2007 - 7467

Aun así, vale acotar que para un grupo de alumnos no es divertido hablar con otros de estadística fuera de la escuela.

Por último, se puede señalar que los estudiantes perciben que la Estadística es demasiado teórica como para ser de utilidad práctica en lo profesional. En síntesis, se puede concluir que la percepción de Tener que utilizar poco la estadística en mi vida profesional es ligeramente alta. Por eso, existe la necesidad de trabajar la actitud hacia la estadística de estos estudiantes mediante una estrategia de aprendizaje adecuada que sirva para motivar y apoyar el rendimiento educativo.

En cuanto a las preguntas formuladas para determinar el nivel de conocimiento teórico adquirido luego de desarrollar el curso de estadística, se puede señalar que los porcentajes y frecuencias de "respuestas acertadas" son satisfactorios, pues en las preguntas 1, 2, 3, 4, 6, 7, 8 y 9 se consiguieron aciertos superiores a $60 \%$.

En general, la percepción de los estudiantes frente a este tipo de estrategia pedagógica de aprendizaje es muy buena, aunque se deben considerar los demás factores de aula (cansancio, pereza, etc.) para alcanzar una mejor ejecución de las actividades.

\section{Futuras líneas de Investigación}

A partir de las evidencias obtenidas, han surgido diversos interrogantes que pueden considerarse en futuros estudios aportar una mejor comprensión de la problemática que se abordó. Es necesario desarrollar estudios que consideren con mayor profundidad cada uno de los factores que influyen en la actitud negativa hacia el aprendizaje de la estadística en los estudiantes.

La evidencia derivada del análisis de las actitudes positivas y negativas que mostraron tener los estudiantes, puede tener alguna relación con las actitudes que tienen los alumnos hacia el docente. De la misma manera, es necesario hacer estudios sobre factores que influyen en el aprendizaje del alumno, que parecen tener una influencia importante en la locomoción de los conocimientos hacia la estadística. 


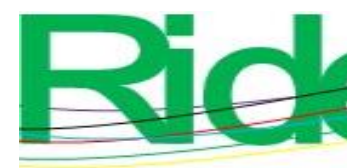

Revista Iberoamericana para la Investigación y el Desarrollo Educativo ISSN $2007-7467$

\section{Referencias}

Astorga, M. A., Flores, B. L., Ibarra, J. E., Mariscal, J. y Vizcarra, L. E. (2017). Impacto del aprendizaje basado en proyectos implementado en una empresa escolar de base tecnológica dedicada al desarrollo de Software. Revista Electrónica de Computación, Informática, Biomédica y Electrónica, 4(4), 1-47. Recuperado de http://recibe.cucei.udg.mx/ojs/index.php/ReCIBE/article/view/54.

Auzmendi, E. (1992). Las actitudes hacia la matemática-estadística en las enseñanzas media y universitaria. Características y medición. España: Ed. Mensajero.

Ayuga, E., González, C., Grande, M. A. y Martínez, E. (2012). Diseño de un cuestionario para evaluar conocimientos básicos de estadística de estudiantes del último curso de ingeniería. Formación Universitaria, 5(1), 21-32. Doi: 10.4067/S071850062012000100004.

Batanero, C. y Díaz, C. (2011). Estadística con proyectos. Granada: Universidad de Granada. Recuperado https://www.ugr.es/ batanero/pages/ARTICULOS/Libroproyectos.pdf.

Bautista, A., Morales, M. V., Dórame, L. G. y Peralta, G. (2016). Un estudio sobre las actitudes hacia la estadística en estudiantes universitarios. Epistemus, Ciencia, Tecnología y Salud, 10(20), 48-54. Recuperado de https://sahuarus.mat.uson.mx/index.php/epistemus/issue/view/17

Cardoso, E. O., Cerecedo, M. T. y Ramos, J. R. (2012). Actitudes hacia las matemáticas de los estudiantes de posgrado en administración: un estudio diagnóstico. Revista de Estudios y Experiencias en Educación, 11(22), 81-98. Recuperado de https://www.redalyc.org/pdf/2431/243125410004.pdf.

Comas, C., Martins, J. A., Nascimento, M. M. y Estrada, A. (2017). Estudio de las actitudes hacia la estadística en estudiantes de psicología. Revista Bolema, Róo Claro (SP), 31(57), 479-496. Doi: 10.1590/1980-4415v31n57a23.

Flores, G. y Juárez, E. D. L. (2017). Aprendizaje basado en proyectos para el desarrollo de competencias matemáticas en bachillerato. Revista Electrónica de Investigación Educativa, 19(3), 71-91. Doi: 10.24320/redie.2017.19.3.721.

García, A. y Guerrero, R. S. (2011). Aprendizaje basado en problemas, proyectos globales y web social. Revista Consejo Latinoamericano de Ciencias Sociales, 1-8. Recuperado de 


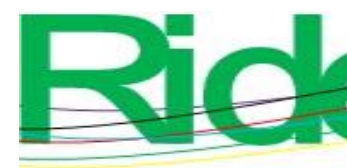

Revista Iberoamericana para la Investigación y el Desarrollo Educativo

ISSN 2007 - 7467

http://biblioteca.clacso.edu.ar/Cuba/cepesuh/20110614104218/aprendizajebasadoen problemas.pdf.

García, A., Escalera, M. E., Moreno, E. and Santana, J del C. (2016). Factors that explains student anxiety toward mathematics. Eurasia Journal of Mathematics, Science and Technology Education, 12(2), 361-372. Doi: 10.12973/eurasia.2016.1216a.

Gil, N., Guerrero, E. y Blanco, L. (2006). El dominio afectivo en el aprendizaje de las Matemáticas. Revista de Investigación Psicoeducativa, 1(4), 47 - 72. Recuperado de https://www.redalyc.org/pdf/2931/293123488003.pdf

Gómez-Chacón, I. M. (2000). Matemática emocional. Los afectos en el aprendizaje matemático. Madrid, España: Narcea, S. A, Ediciones

Hernández, R., Fernández, C. y Baptista, P. (2014). Metodología de la Investigación (6. ${ }^{\text {e ed). }}$ México, D. F.: Mcgraw-Hill / Interamericana Editores, S.A. De C.V.

Inzunza, S. (2017). Potencial de los proyectos para desarrollar motivación, competencias de razonamiento y pensamiento estadístico. Actualidades Investigativas en Educación, 17(3), 1-30. Doi: 10.15517/aie.v17i3.29874.

Izcara, S. P. (2014). Manuel de investigación cualitativa (1. ${ }^{\mathrm{a}}$ ed.). México: Fontamara.

Jonassen, D. H. (1997). Instructional design models for well-structured and III-structured problem-solving learning outcomes. Educational Technology Research and Development, 45(1), 65-94. Retrieved from https://link.springer.com/article/10.1007\%2FBF02299613.

Lind, D. A., Marchal, W. G. y Mason R. D. (2004). Estadística para la administración y economía (11. ${ }^{\mathrm{a}}$ ed.). México D. F: Alfaomega.

Otzen, T. y Manterola, C. (2017). Técnicas de muestreo sobre una población a estudio. International Journal of Morphology, 35(1), 227-232. Doi: 10.4067/S071795022017000100037.

Rincón, W. A. (2014). Preguntas abiertas en encuestas ¿cómo realizar su análisis? Comunicaciones en Estadística, 7(2), 139-156. Doi: 10.15332/s20273355.2014.0002.02.

Rodríguez, E. y Cortés, M. (2010). Evaluación de la estrategia pedagógica "aprendizaje basado en proyectos": percepción de los estudiantes. Revista da Avaliação da Educación Superior, 15(1), 143-158. Doi: 10.1590/S1414-40772010000100008. 


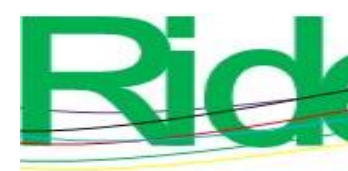

Revista Iberoamericana para la Investigación y el Desarrollo Educativo

ISSN 2007 - 7467

Rodríguez, N. (2011). Actitudes de los estudiantes universitarios hacia la estadística. Revista Psicológicas y Ciencias Afines, Interdisciplinaria, 28(2), 199-205. Recuperado de https://www.redalyc.org/pdf/180/18022339002.pdf

Rojas, C. A., Escalera, M. E., Moreno, E. y García, A. (2017). Motivación, ansiedad, confianza, agrado y utilidad. Los factores que explican la actitud hacia las matemáticas en los estudiantes de Economía. Revista INFAD de Psicología. International Journal of Developmental and Educational Psychology, 2(1), 527-540. Doi: 10.17060/ijodaep.2017.n1.v2.875.

Salinas, J. y Mayén, S. (2016). Estudio exploratorio de las actitudes hacia la estadística en estudiantes mexicanos de bachillerato. Avances de Investigación en Educación Matemática, (10), 73-90. Doi: 10.35763/aiem.v0i10.130

Sánchez, A. I. (2019). Identificación de actitudes hacia la estadística en estudiantes de la licenciatura en Matemáticas (tesis de maestría). Universidad Autónoma de Zacatecas. Recuperado de http://ricaxcan.uaz.edu.mx/jspui/bitstream/20.500.11845/1360/1/2019\%20S\%C3\% A1nchez\%20A.pdf.

Schau, C. (2003). Students' attitudes: The "other" important outcome in statistics education. Proceedings of the joint statistical meetings (3673-3681). Retrieved from http://citeseerx.ist.psu.edu/viewdoc/download?doi=10.1.1.231.6258\&rep=rep1\&typ $\mathrm{e}=\mathrm{pdf}$

Toledo, G., Arellano, J. J., Aguilar, F. y Molina, E. W. (2018). Aprendizaje basado en proyectos dentro de un curso universitario de interacción humano computadora. Revista Electrónica de Computación, Informática, Biomédica y Electrónica, 7(2), 6591. Recuperado de http://recibe.cucei.udg.mx/ojs/index.php/ReCIBE/article/view/101.

Torres, F., Aparicio, A. S., Bazán, J. L. y Abdounur, O. J. (2015). Actitudes hacia la estadística en universitarios del área de las ciencias de una universidad pública de Chile. Educación Matemática Pesquisa, 17(1), 45-73. Recuperado de https://repositorio.usp.br/bitstream/handle/BDPI/51337/2713161.pdf?sequence=2. 


\begin{tabular}{|c|c|}
\hline Rol de Contribución & Autor (es) \\
\hline Conceptualización & Silberio García Sánchez (principal) \\
\hline Metodología & $\begin{array}{l}\text { Silberio García Sánchez (principal), Alejandro Juárez Agis } \\
\text { (apoya) }\end{array}$ \\
\hline Software & No aplica. \\
\hline Validación & $\begin{array}{l}\text { Silberio García Sánchez (principal), Alejandro Juárez Agis } \\
\text { (igual), Branly Olivier Salomé (igual), Jacqueline Zeferino } \\
\text { Torres (igual), Mayra Rivas González (igual). }\end{array}$ \\
\hline Análisis Formal & $\begin{array}{l}\text { Silberio García Sánchez (principal), Alejandro Juárez Agis } \\
\text { (igual), Branly Olivier Salomé (igual). }\end{array}$ \\
\hline Investigación & $\begin{array}{l}\text { Silberio García Sánchez (principal), Alejandro Juárez Agis } \\
\text { (igual), Branly Olivier Salomé (igual), Jacqueline Zeferino } \\
\text { Torres (igual), Mayra Rivas González (igual). }\end{array}$ \\
\hline Recursos & $\begin{array}{l}\text { Silberio García Sánchez (principal), Alejandro Juárez Agis } \\
\text { (igual), Branly Olivier Salomé (igual), Jacqueline Zeferino } \\
\text { Torres (igual), Mayra Rivas González (igual). }\end{array}$ \\
\hline Curación de datos & $\begin{array}{l}\text { Silberio García Sánchez (principal), Alejandro Juárez Agis } \\
\text { (igual), Branly Olivier Salomé (igual), Jacqueline Zeferino } \\
\text { Torres (igual), Mayra Rivas González (igual). }\end{array}$ \\
\hline $\begin{array}{l}\text { Escritura - Preparación del } \\
\text { borrador original }\end{array}$ & Silberio García Sánchez (principal) \\
\hline $\begin{array}{l}\text { Escritura - Revisión y } \\
\text { edición }\end{array}$ & $\begin{array}{l}\text { Silberio García Sánchez (principal), Alejandro Juárez Agis } \\
\text { (igual), Branly Olivier Salomé (igual), Jacqueline Zeferino } \\
\text { Torres (igual), Mayra Rivas González (igual). }\end{array}$ \\
\hline Visualización & $\begin{array}{l}\text { Silberio García Sánchez (principal), Alejandro Juárez Agis } \\
\text { (igual), Branly Olivier Salomé (igual), Jacqueline Zeferino } \\
\text { Torres (igual), Mayra Rivas González (igual). }\end{array}$ \\
\hline Supervisión & $\begin{array}{l}\text { Silberio García Sánchez (principal), Alejandro Juárez Agis } \\
\text { (igual), Branly Olivier Salomé (igual), Jacqueline Zeferino } \\
\text { Torres (igual), Mayra Rivas González (igual). }\end{array}$ \\
\hline $\begin{array}{l}\text { Administración de } \\
\text { Proyectos }\end{array}$ & $\begin{array}{l}\text { Silberio García Sánchez (principal), Alejandro Juárez Agis } \\
\text { (igual), Branly Olivier Salomé (igual), Jacqueline Zeferino } \\
\text { Torres (igual), Mayra Rivas González (igual). }\end{array}$ \\
\hline Adquisición de fondos & $\begin{array}{l}\text { Silberio García Sánchez (principal), Alejandro Juárez Agis } \\
\text { (igual), Branly Olivier Salomé (igual), Jacqueline Zeferino } \\
\text { Torres (igual), Mayra Rivas González (igual). }\end{array}$ \\
\hline
\end{tabular}

